\title{
Network electro-thermal simulation of non- isothermal magnetohydrodynamic heat transfer from a transpiring cone with buoyancy and pressure work.
}

BÉG, O.A., ZUECO, J., KADIR, A., BÉG, T.A. and KHAN, U.F.

This is a post-peer-review, pre-copyedited version of an article published in International Journal of Applied and Computational Mathematics. The final authenticated version is available online at: https:// doi.org/10.1007/s40819-016-0192-5. This pre-copyedited version is made available under the Springer terms of reuse for AAMs: https://www.springer.com/gp/open-access/publication-policies/aam-terms-of-use 


\title{
NETWORK ELECTRO-THERMAL SIMULATION OF NON-ISOTHERMAL MAGNETOHYDRODYNAMIC HEAT TRANSFER FROM A TRANSPIRING CONE WITH BUOYANCY AND PRESSURE WORK
}

\author{
O. Anwar Bég* \\ Fluid Mechanics, Spray Research Group, Petroleum/Gas Engineering Division, School of Computing, \\ Science \& Engineering, G77, Newton Building, University of Salford, Manchester, M54WT, England, UK. \\ J. Zueco \\ Departamento de Ingeniería Térmica y Fluidos, Univ. Politécnica de Cartagena, Murcia, Spain. \\ Ali Kadir \\ Spray Research Group, Petroleum/Gas Engineering Division, School of Computing, Science and \\ Engineering, G79, Newton Building, University of Salford, Manchester, M54WT, England, UK. \\ Tasveer A. Bég \\ Renewable Energy and Geodynamics Research, Dickenson Road, Manchester, England, UK. \\ Umar F. Khan \\ Magnetic Materials Research, Electrical/Electronic Engineering, Huddersfield University, England, UK.
}

\begin{abstract}
The steady, axisymmetric laminar natural convection boundary layer flow from a non-isothermal vertical circular porous cone under a transverse magnetic field, with the cone vertex located at the base, is considered. The pressure work effect is included in the analysis. The governing boundary layer equations are formulated in an (x,y) coordinate system (parallel and normal to the cone slant surface), and the magnetic field effects are simulated with a hydromagnetic body force term in the momentum equation. A dimensionless transformation is performed rendering the momentum and also heat conservation equations. The thermal convection flow is shown to be controlled by six thermophysical parameters- local Hartmann number, local Grashof number, pressure work parameter, temperature power law exponent, Prandtl number and the transpiration parameter. The transformed parabolic partial differential equations are solved numerically using the Network Simulation Method (NSM) based on the electrical-thermodynamic analogy. Excellent correlation of the zero Hartmann number case is achieved with earlier electrically nonconducting solutions. Local shear stress function (skin friction) is found to be strongly decreased with an increase in Prandtl number (Pr), with negative values (corresponding to flow reversal) identified for highest Pr with further distance along the streamwise direction. A rise in local Hartmann number, is observed to depress skin friction. Increasing temperature power law index, corresponding to steeper temperature gradient at the wall, strongly reduces skin friction at the cone surface. A positive rise in pressure work parameter decreases skin friction whereas a negative increase elevates the skin friction for some distance along the cone surface from the apex. Local heat transfer gradient is markedly boosted with a rise in Prandtl number but decreased principally at the cone surface with increasing local Hartmann number. Increasing temperature power law index conversely increases the local heat transfer gradient, at the cone surface. A positive rise in pressure work parameter increases local heat transfer gradient while negative causes it to decrease. A rise in local Grashof number boosts local skin friction and velocity into the boundary layer; local heat transfer gradient is also increased with a rise in local Grashof number whereas the temperature in the boundary layer is noticeably reduced. Applications of the work arise in spacecraft magnetogas dynamics, chemical cooling systems and industrial magnetic materials processing.
\end{abstract}

Key words: Magnetofluid dynamics; Numerical Solutions; Prandtl number; Hartmann number; Pressure work; Non-Isothermal; Lateral Mass Flux; Heat Transfer; local Nusselt number; Nonlinear convection.

* Author to whom correspondence should be addressed; Email-O.A.Beg@salford.ac.uk; gortoab@gmail.com. 


\section{INTRODUCTION}

The ever-increasing interest in understanding better the aero-thermodynamics and near-field heat transfer in astronautical bodies such as spacecraft, space modules, satellites etc has continued to stimulate significant research efforts. Of the geometries analyzed, spherical bodies, cones and wedge configurations have received special attention. Convective heat transfer is important in tubular thrust rocket chambers [1], rocket nozzle design [2] and various aspects of industrial manufacturing technologies [3]. Bartz [4] reported on convection heat transfer from a rocket nozzle. Streif [5] studied experimentally the heat transfer to a cone with a 70 degrees apex angle at several angles of attack in a wind tunnel at free stream Mach number of 7 . He obtained good correlation with conical flow theory. Braun et al [6] used an integral method to study the isothermal free convection similarity flows about two-dimensional and axisymmetric bodies over a wide spectrum of Prandtl. Hering and Grosh [7] showed that boundary layer similarity solutions are only possible for convection from a cone when the surface temperature variation is a power function of distance along a cone ray. Isothermal solutions were also presented as were linear temperature variation solutions for Prandtl number of 0.7. Hering [8] subsequently extended this work to consider lower Prandtl number cases. Roy [9] presented a similar model to Hering [8], for the case of high Prandtl numbers. Na and Chiou [10] over a decade later discussed the effect of slenderness i.e. conical geometry, on free convection flow, with applications in rocket nose aerodynamic heat transfer. Lin [11] examined the isothermal case for large cone angles where boundary layer thickness is small in comparison with the local cone radius, neglecting therefore transverse curvature effects. Alamgir [12] has employed an integral method to investigate the effective heat transfer rates in free convection laminar boundary layers on a vertical cone. Pop and Takhar [13] have studied compressibility effects in laminar conical body free convection using shooting numerical methods. More recent studies of the convection from a cone have been reported by Hossain et al [14] who studied temperature-dependent viscosity effects. Hossain and Paul [15] studied transpiration effects and later Takhar et al [16] analyzed numerically the influence of thermophysical property variation on gas convective heat transfer over a vertical cone. More recently Chamkha et al [17] have studied computationally the effect of unsteadiness and also compressibility on a cone. Very recently Roy et al [18] have re-examined the transient convection boundary layer flow over a cone with transpiration effects. Singh and Roy [19] have also studied the development of unsteady mixed convection flow of an incompressible laminar viscous fluid over a vertical cone with the fluid in the external stream set into motion impulsively, and a simultaneous sudden surface temperature. The problem was shown to reduce at $t=0$, to a Rayleigh type of equation and as $t \rightarrow \infty$, to a Falkner-Skan type of equation. The scale of time 
has been selected such that the traditional infinite region of integration become finite which significantly reduce the computational time. Using the quasi-linearization technique they showed that there is a smooth transition from the initial steady state to the final steady state. Kumari, and Nath [20] have considered the unsteady laminar compressible boundary layer over a circular cone at an angle of attack near a plane of symmetry in hypersonic flow. The case of the boundary layer near the windward and leeward planes has been considered with suction effects. The effects of the variable fluid properties, non-unity Prandtl number and viscous dissipation were considered. Computations showed that in a small time interval immediately after the start of the impulsive motion, the direction of the heat transfer changes. The surface shear stresses in the streamwise and cross-wise directions and the surface heat transfer, increased with time and attained the final steady state values rapidly i.e. small spin-up times were reported. The total enthalpy at the wall was shown to considerably influence the surface shear stresses in the streamwise and cross-flow directions and the surface heat transfer. Suction was found to strongly affect the surface shear stress in the streamwise direction and the surface heat transfer. These studies were all confined to electrically non-conducting fluids. However due to the ionized nature of air in hypersonic flows and astronautical transport the flow can become electrically-conducting i.e. hydromagnetic. At weak magnetic fields basically a Lorentzian drag force is imparted to the flow field; at higher magnetic field strengths, ionslip and Hall currents also arise. Magnetogas dynamics has therefore received considerable attention in the aerospace engineering community for several decades. An excellent treatise on the subject is available in Pai [21]. Motivated largely by high temperature processes involved in space craft re-entry and interaction with planetary magnetospheric layers, engineers have considered a number of magneto-gas convection flows past various geometrical configurations. Sluyter and Touryan [21] studied the magnetohydrodynamic (MHD) compressible boundary layer flow on a rotating cone. Surma Devi et al [22] investigated the combined effects of the magnetic field, mass transfer, and heat transfer on the steady incompressible laminar boundary-layer flow of an electrically conducting fluid over a non-isothermal cone using a shooting method. The magnetic field or injection were shown to reduce both the skin friction and heat transfer, with the converse behaviour exhibited for the effect of suction. Heat transfer was also shown to be increased for higher Prandtl numbers. The influence of a radial magnetic field on MHD flow between a cone and cylinder rotating co-axially was reported by Singh et al [23]. Further studies on hydromagnetic convection from a cone have been reported in the context of industrial and geophysical processes by Chamkha [24], Chamkha et al. [25], Chamkha [26] and more recently by Chamkha and Al-Mudhaf [27]. These studies did not consider the important effect of pressure work or viscous dissipation. Alam et al [28] very recently utilized the Gebhart 
formulation [29] for pressure work effect in natural convection from a porous cone using the Keller-box numerical method. Takhar and Soundalgekar [30] studied viscous heating and stress work effects on boundary layer convection using a shooting scheme. Several studies have also appeared concentrating on the influence of non-isothermal surface temperature variation, although not in the context of boundary layer convection from a cone. Of the important studies conducted we mention here the analysis by Soundalgekar et al [31], Soundalgekar et al [32] who considered transient stagnation point hydromagnetic convection flow with non-isothermal effects and Ganesan et al [33]. In the present analysis we shall consider the hydromagnetic gas convection flow past a circular cone geometry (e.g. spacecraft nose), with significant pressure work effects and non-isothermal surface temperature variation. Such a study has thusfar not been communicated in the literature despite important applications in spacecraft magneto-gas dynamic heat transfer. A transformed version of the conservation equations is solved numerically. The influence of surface transpiration (of importance in cooling astronautical bodies during re-entry), local Hartmann hydromagnetic number, Prandtl number, local Grashof number, non-isothermal parameter and pressure work parameter on surface skin friction and Nusselt number are computed and described graphically.

\section{MATHEMATICAL MODEL}

The physical problem to be investigated is shown in Figure 1.

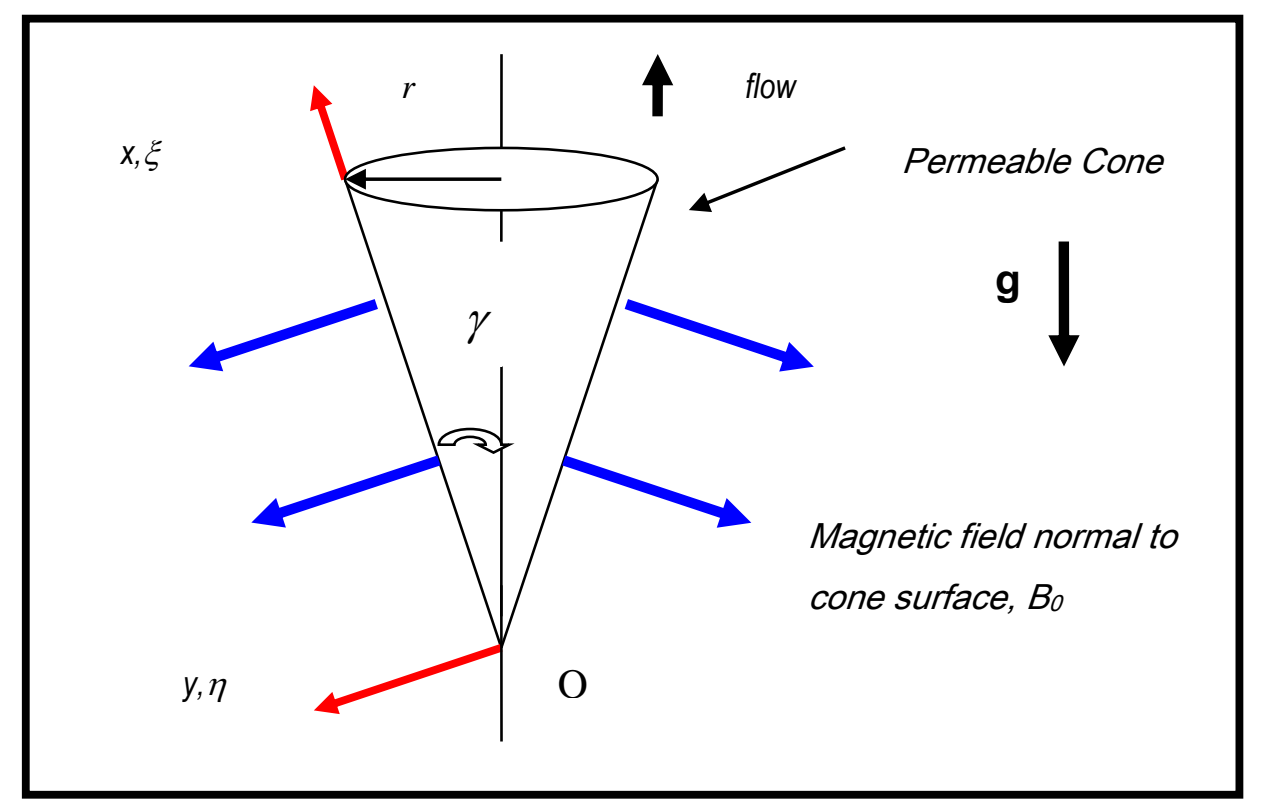

Figure 1: Physical model for magnetohydrodynamic heat transfer from a cone

We consider the steady, two-dimensional, laminar, incompressible, axisymmetric, hydromagnetic free convection boundary layer flow of an electrically-conducting gas external to a porous, non- 
isothermal vertical circular cone in a Cartesian $(x, y)$ coordinate system. The $x$-direction is measured along the cone surface from the leading edge, $O$. A uniform magnetic field is applied perpendicular to the cone surface. For laminar, incompressible, electrically-conducting, Newtonian fluids subjected to a transverse magnetic field, the fluid is known to experience a retarding force. The motivation of the present study is to elucidate the interaction of magnetic force with thermal and aerodynamic velocity boundary layers along the entire geometry of the cone surface. The Maxwell field equations, as described by Landau and Lifschitz [34] comprise five vector equations- the Ampere law, magnetic field continuity, Faraday's law, Kirchoff's law and finally Ohm's law. The generalized equations in vectorial form, for flow of an electricallyconducting gas are the Maxwell equations:

$$
\begin{array}{ll}
\nabla \times \mathbf{B}=\mu \mathbf{J} & \text { Ampere's Law } \\
\nabla \bullet \mathbf{B}=0 & \text { Magnetic Field Continuity (Maxwell Equation) } \\
\nabla \times \mathbf{E}=-\frac{\partial \mathbf{B}}{\partial t} & \text { Faraday's Law } \\
\nabla \bullet \mathbf{J}=0 & \text { Kirchoff's Law } \\
\mathbf{J}=\sigma[\mathbf{E}+v \times \mathbf{B}] \text { Ohm's Law }
\end{array}
$$

where $\boldsymbol{J}$ is the current density, $\boldsymbol{B}$ is the magnetic field vector, $\sigma$ is the electrical conductivity, $\boldsymbol{E}$ is the electrical field intensity vector, $\rho$ is density, $v$ is the velocity vector, $\mu$ is viscosity, $t$ is time. From an order of magnitude analysis, it can be shown [34] that for two-dimensional $(x-y)$ magneto-hydrodynamic gas dynamic flows, the hydromagnetic retarding force (Lorentz body force) acts only parallel to the flow and has the form:

$$
F_{\text {magnetic }} \approx-\sigma B_{y}^{2} u
$$

where $B_{y}$ is the component of magnetic field in the $y$-direction. We consider an aerodynamic viscous flow where the magnetic field is sufficiently weak to sustain a small magnetic Reynolds number such that induced magnetic field effects can be neglected. Joule electro-heating and Hall 
current/ionslip effects are also neglected. Pressure work effects are however significant and included. The $x$-direction is parallel to the cone slant surface, the $y$-direction normal to this. The governing boundary layer equations for the steady, axisymmetric, electrically-conducting gas flow regime can be posed as follows with reference to the $(x, y)$ coordinate system:

Conservation of Mass:

$$
\frac{\partial(u r)}{\partial x}+\frac{\partial(v r)}{\partial y}=0
$$

Conservation of Momentum

$$
u \frac{\partial u}{\partial x}+v \frac{\partial u}{\partial y}=v \frac{\partial^{2} u}{\partial y^{2}}+g \beta \operatorname{Cos} \gamma\left(T-T_{\infty}\right)-\frac{\sigma_{0} B_{0}{ }^{2}}{\rho} u
$$

\section{Conservation of Thermal Energy (Heat):}

$$
u \frac{\partial T}{\partial x}+v \frac{\partial T}{\partial y}=\alpha \frac{\partial^{2} T}{\partial Y^{2}}+\frac{T \beta u}{\rho c_{p}} \frac{\partial p}{\partial x}
$$

where $u, v$ are the velocity components in the $x$ - and $y$-directions, $v$ is kinematic viscosity, $g$ is gravitational acceleration, $\alpha$ is thermal diffusivity of the gas, $\beta$ is the coefficient of volume expansion, $T$ is fluid temperature, $\gamma$ is cone apex half angle, $c_{p}$ is the isobaric specific heat of the gas, $\rho$ is density of the gas, $\sigma$ is electrical conductivity of the gas and $B_{0}$ is magnetic field. The Boussinesq approximation has been used so that buoyancy effects only appear in the $x$-direction momentum equation, which is coupled to the energy equation, constituting a free convection regime. Viscous dissipation effects are neglected. The corresponding boundary conditions at the surface and far from the cone are, following Hossain et al. [14]:

$$
\begin{aligned}
& u=0, v=-V, T=T_{w}(x) \text { at } y=0 . \\
& u=0, T=T_{\infty} \text { as } y \rightarrow \infty .
\end{aligned}
$$

where $T_{W}$ is the cone surface temperature $\left(>T_{\infty}\right), T_{\infty}$ is the ambient fluid temperature, $V$ denotes transpiration velocity of fluid via the cone surface (lateral mass flux velocity). For $V>0$, suction through the cone surface from the boundary layer occurs i.e. gas removal. With $V<0$ injection i.e. blowing of gas into the boundary layer occurs. We consider both cases and also the case of a solid cone surface i.e. $V=0$, in the present study. The equations (7) to (9) are highly coupled, 
parabolic and nonlinear. An analytical solution is clearly intractable and in order to facilitate a numerical solution we non-dimensionalize the model.

\section{TRANSFORMATION OF MAGNETO-CONVECTIVE MODEL EQUATIONS}

We now introduce the following transformations:

$$
\begin{aligned}
& \xi=\frac{V x}{v}\left[G r_{x}\right]^{1 / 4} \\
& \eta=\frac{y}{x}\left[G r_{x}\right]^{1 / 4} \\
& r=x \sin \gamma \\
& \psi=v r\left[G r_{x}\right]^{1 / 4}\left[F(\xi, \eta)+\frac{1}{2} \xi\right] . \\
& G(\xi, \eta)=\frac{T-T_{\infty}}{T_{w}-T_{\infty}} . \\
& G r_{x}=\frac{g \beta \cos \gamma\left[T_{w}-T_{\infty}\right] x^{3}}{v^{2}} . \\
& T_{w}-T_{\infty}=x^{n} \\
& \operatorname{Pr}=\frac{v}{\alpha} \\
& H a_{x}=\sqrt{\frac{\sigma}{\mu}} B_{o} x \\
& \varepsilon=\frac{g \beta x}{c_{p}} \\
& u=\frac{1}{r} \frac{\partial \psi}{\partial y} \\
& v=-\frac{1}{r} \frac{\partial \psi}{\partial x}
\end{aligned}
$$

where $\xi$ is the streamwise coordinate (dimensionless transpiration parameter), $\eta$ is the spanwise pseudo-similarity coordinate, $\psi$ is stream function, $r$ is radial coordinate, $G(\xi, \eta)$ is dimensionless temperature function, $F(\xi, \eta)$ is dimensionless stream function, $G r_{x}$ is the local Grashof number, $n$ is the temperature power law exponent, $P r$ is Prandtl number, $H a_{x}$ is local Hartmann number and $\varepsilon$ is the pressure work parameter. Eqns. (22) and (23) are the Cauchy-Riemann equations which 
identically satisfy the conservation of mass equation (7). Implementing these transformations in the conservation equations (7) to (9), we arrive at the following dimensionless nonsimilar partial differential equations:

$$
\begin{aligned}
& \frac{\partial^{3} F}{\partial \eta^{3}}+\frac{(n+7)}{4} F \frac{\partial^{2} F}{\partial \eta^{2}}-\frac{(n+1)}{2}\left[\frac{\partial F}{\partial \eta}\right]^{2}+G+\xi \frac{\partial^{2} F}{\partial \eta^{2}}=\frac{(1-n)}{4} \xi\left[\frac{\partial F}{\partial \eta} \frac{\partial^{2} F}{\partial \xi \partial \eta}-\frac{\partial^{2} F}{\partial \eta^{2}} \frac{\partial F}{\partial \xi}\right]+ \\
& +\frac{H a_{x}^{2}}{G r_{x}^{1 / 2}} \frac{\partial F}{\partial \eta} \\
& \frac{1}{\operatorname{Pr}} \frac{\partial^{2} G}{\partial \eta^{2}}+\frac{(n+7)}{4} F \frac{\partial G}{\partial \eta}-(n+\varepsilon) G \frac{\partial F}{\partial \eta}+\xi \frac{\partial G}{\partial \eta}=\frac{(1-n)}{4} \xi\left[\frac{\partial F}{\partial \eta} \frac{\partial G}{\partial \xi}-\frac{\partial G}{\partial \eta} \frac{\partial F}{\partial \xi}\right]
\end{aligned}
$$

The transformed boundary conditions now become:

At the cone surface

$$
\eta=0: \quad F=\frac{\partial F}{\partial \eta}=0 ; G=1
$$

In the free stream;

$$
\eta \rightarrow \infty: \quad \frac{\partial F}{\partial \eta}=0 ; G=0
$$

We note that while the present equations are valid for free convection, setting $G r_{x} \rightarrow 1$ reduces the system to a mixed convection regime. We seek a full numerical solution to the transformed local non-similar partial differential equations (24) and (25) under the boundary conditions (26) and (27). Of importance in rocket (and other areas of technological) heat transfer are several key design parameters based on the differentials of the velocity and temperature functions, $F$ and $G$. Using a reference velocity, $U\left(=v[G r]^{1 / 2} / x\right)$, the local skin friction coefficient, $C_{f x}$, is defined as:

$$
C_{f x}=\frac{\tau_{w}}{\rho U^{2}}=\frac{\mu \nu \frac{\left[G r_{x}\right]^{3 / 4}}{x^{2}} \frac{\partial^{2} F(\xi, 0]}{\partial \eta^{2}}}{\frac{\rho v^{2} G r_{x}}{x^{2}}}=\frac{\frac{\partial^{2} F(\xi, 0]}{\partial \eta^{2}}}{G r_{x}^{1 / 4}}
$$

The local Nusselt number, $N u_{x}$, takes the form: 


$$
N u_{x}=-\frac{q_{w} x}{\kappa\left[T_{w}-T_{\infty}\right]}=-\frac{-\kappa \frac{x^{n} G r_{x}{ }^{1 / 4}}{x} \frac{\partial G(\xi, 0)}{\partial \eta} x}{\kappa\left[T_{w}-T_{\infty}\right]}=-G r_{x}{ }^{1 / 4} \frac{\partial G(\xi, 0)}{\partial \eta}
$$

\section{SPECIAL CASES OF THE FLOW MODEL}

Let us briefly concentrate now on a number of special cases which can be retrieved from the general flow model discussed in section 3 .

\section{Case I: Non-magnetic Non-Isothermal Gas Convection from Permeable Cone}

Setting $H a_{x} \rightarrow O$ eliminates the Lorentz body force term due to the magnetic field and reduces the momentum equation to the case considered by Alam et al [28].

$$
\frac{\partial^{3} F}{\partial \eta^{3}}+\frac{(n+7)}{4} F \frac{\partial^{2} F}{\partial \eta^{2}}-\frac{(n+1)}{2}\left[\frac{\partial F}{\partial \eta}\right]^{2}+G+\xi \frac{\partial^{2} F}{\partial \eta^{2}}=\frac{(1-n)}{4} \xi\left[\frac{\partial F}{\partial \eta} \frac{\partial^{2} F}{\partial \xi \partial \eta}-\frac{\partial^{2} F}{\partial \eta^{2}} \frac{\partial F}{\partial \xi}\right]
$$

The energy equation (24) and boundary conditions (26) and (27) are unchanged. One objective of the present study is to investigate the interaction of Hartmann number with the suction parameter, $\xi$. Blowing i.e. $\xi<0$ has been implemented in many practical rocket/space craft designs in liquid propellant systems. For example sweat cooling involves the injection of fluid or gas via porous walls. Alternatively film cooling utilizes the presence of an injection pattern providing a layer of relatively cool gas in the wall vicinity which serves to significantly reduce heat transfer at the surface [35]. Blowing however is not considered here for brevity.

\section{Case II: Non-magnetic Non-Isothermal Gas Convection from Solid Cone}

Setting $\xi \rightarrow 0$ reduces our general model to the one-dimensional model considered in a classical study by Hering and Grosh [7]. In this case, equations (24) and (25) reduce to:

$$
\begin{aligned}
& \frac{\partial^{3} F}{\partial \eta^{3}}+\frac{(n+7)}{4} F \frac{\partial^{2} F}{\partial \eta^{2}}-\frac{(n+1)}{2}\left[\frac{\partial F}{\partial \eta}\right]^{2}+G=0 \\
& \frac{1}{\operatorname{Pr}} \frac{\partial^{2} G}{\partial \eta^{2}}+\frac{(n+7)}{4} F \frac{\partial G}{\partial \eta}-(n+\varepsilon) G \frac{\partial F}{\partial \eta}=0
\end{aligned}
$$

Infact for this one-dimensional case, a simple Runge-Kutta-Merson shooting algorithm or a finite element "line" model would provide robust solutions. However we have elected to use NSM in the present study. 


\section{Case III: Non-magnetic Isothermal Gas Convection from Permeable Cone}

Setting $n \rightarrow 0$ (with $H a_{x}=0$ ) reduces the general model to the two-dimensional $(\xi, \eta)$ isothermal cone surface case, considered by $\mathrm{Na}$ and Chiou [9]. In this case, the momentum and energy equations (24) and (25) simplify to:

$$
\begin{aligned}
& \frac{\partial^{3} F}{\partial \eta^{3}}+\frac{7}{4} F \frac{\partial^{2} F}{\partial \eta^{2}}-\frac{1}{2}\left[\frac{\partial F}{\partial \eta}\right]^{2}+G+\xi \frac{\partial^{2} F}{\partial \eta^{2}}=\frac{1}{4} \xi\left[\frac{\partial F}{\partial \eta} \frac{\partial^{2} F}{\partial \xi \partial \eta}-\frac{\partial^{2} F}{\partial \eta^{2}} \frac{\partial F}{\partial \xi}\right] \\
& \frac{1}{\operatorname{Pr}} \frac{\partial^{2} G}{\partial \eta^{2}}+\frac{7}{4} F \frac{\partial G}{\partial \eta}-\varepsilon G \frac{\partial F}{\partial \eta}+\xi \frac{\partial G}{\partial \eta}=\frac{1}{4} \xi\left[\frac{\partial F}{\partial \eta} \frac{\partial G}{\partial \xi}-\frac{\partial G}{\partial \eta} \frac{\partial F}{\partial \xi}\right]
\end{aligned}
$$

Case IV: Mixed Non-magnetic Isothermal Gas Convection from Permeable Cone without Pressure Work

Setting $G r_{x} \rightarrow 1$ renders the viscous hydrodynamic force in the boundary layer of the same order as the buoyancy force and the flow becomes a mixed convection regime. The $\xi$ coordinate will

therefore reduce to $\xi=\frac{V x}{v}$ in equations (31) and (32). Also with $\varepsilon \rightarrow 0$, pressure work effects vanish. This reduces the energy equation (34) to:

$$
\frac{1}{\operatorname{Pr}} \frac{\partial^{2} G}{\partial \eta^{2}}+\frac{7}{4} F \frac{\partial G}{\partial \eta}+\xi \frac{\partial G}{\partial \eta}=\frac{1}{4} \xi\left[\frac{\partial F}{\partial \eta} \frac{\partial G}{\partial \xi}-\frac{\partial G}{\partial \eta} \frac{\partial F}{\partial \xi}\right]
$$

\section{NSM NUMERICAL SOLUTIONS}

NSM has been used extensively in many areas of heat transfer, fluid dynamics and multi-physical transport phenomena [35,36]. To solve the set of non-linear differential equations (24-25) subject to boundary condition (26-27), the Network Simulation Method is very suitable. The principal advantage of NSM is that it can accommodate any type of non-linearity into the model whether in terms of boundary conditions, convective terms, coupled (buoyancy) terms, nonlinear acceleration terms, shear terms, phase-change processes, temperaturedependencies of the thermal properties and so on. It also involves considerably less laborious algebra than standard finite difference or finite element methods. NSM further allows a good analogy with thermo-electrics since it permits easy representation of complex thermal and fluid terms via resistors, capacitors and non-linear devices that seek to resemble thermal systems governed by unsteady linear or non-linear equations. NSM 
is also equally versatile at solving both unsteady and steady-state problems and has a powerful advantage in that programming of the code (Pspice) avoids the conventional pathway of other numerical methods which require manipulation of the sophisticated mathematical software. The third-order differential equation (24) is converted to second-order by substituting $H=F^{\prime}$. Subsequently all second-order equations for $H$ and $G$ are discretized using three-point central difference quotients while the first-order differential equation $H=F^{\prime}$ is discretized by the trapezoidal rule. The equations (24-25) may therefore be re-written as:

$$
\begin{aligned}
& \frac{\partial^{2} H}{\partial \eta^{2}}+\frac{(n+7)}{4} F \frac{\partial H}{\partial \eta}-\frac{(n+1)}{2} H^{2}+G+\xi \frac{\partial H}{\partial \eta}=\frac{(1-n)}{4} \xi\left[H \frac{\partial H}{\partial \xi}-\frac{\partial H}{\partial \eta} \frac{\partial F}{\partial \xi}\right]+\frac{H a_{x}^{2}}{G r_{x}^{1 / 2}} H \\
& \frac{1}{\operatorname{Pr}} \frac{\partial^{2} G}{\partial \eta^{2}}+\frac{(n+7)}{4} F \frac{\partial G}{\partial \eta}-(n+\varepsilon) G H+\xi \frac{\partial G}{\partial \eta}=\frac{(1-n)}{4} \xi\left[H \frac{\partial G}{\partial \xi}-\frac{\partial G}{\partial \eta} \frac{\partial F}{\partial \xi}\right]
\end{aligned}
$$

In NSM the starting point is the set of ordinary differential equations, one for each control volume, obtained either by spatial discretization of the equations (36-37). Based on these equations, a network circuit is designed, whose equations are formally equivalent to the discretized ones. The variables, $F, H$ and $G$ are equivalent to the variable voltage, and their derivates are equivalent to the electric current. A sufficient number of networks are connected in series to constitute the whole medium and boundary conditions are added by means of special electrical devices. The whole network must be coded in an adequate program that can be solved by a computer code. The cases studied here were solved by the software code Pspice [37] using a PC. The following currents are defined:

$$
\begin{aligned}
j_{H, \eta} & \equiv \frac{d H}{d \eta} \\
j_{G, \eta} & \equiv \frac{d G}{d \eta} \\
j_{F, \xi} & =\frac{\partial F}{\partial \xi} \\
j_{H, \xi} & =\frac{\partial H}{\partial \xi} \\
j_{G, \xi} & =\frac{\partial G}{\partial \xi}
\end{aligned}
$$


With these definitions of the currents, the dimensionless equations of momentum, and energy may be postulated in the following form:

$$
\begin{aligned}
& \frac{d j_{H, \eta}}{d \eta}+\frac{(n+7)}{4} F j_{H, \eta}-\frac{(n+1)}{2} H^{2}+G+\xi j_{H, \eta}=\frac{(1-n)}{4} \xi\left[H j_{H, \xi}-j_{H, \eta} j_{F, \xi}\right]+\frac{H a_{x}^{2}}{G r_{x}{ }^{1 / 2}} H \\
& \frac{1}{\operatorname{Pr}} \frac{d j_{G, \eta}}{d \eta}+\frac{(n+7)}{4} F j_{G, \eta}-(n+\varepsilon) G H+\xi j_{G, \eta}=\frac{(1-n)}{4} \xi\left[H j_{G, \xi}-j_{G, \eta} j_{F, \xi}\right]
\end{aligned}
$$

With spatial discretization of the dimensionless equations in $N_{\eta}$ cells of length $\Delta \eta=\eta_{\max } / N_{\eta}$, with $N_{\eta}=150$ in the $\eta$-direction and of $N_{\xi}$ cells of length $\Delta \xi=\xi_{\max } / N_{\xi}$ in the $\xi$-direction with $N_{\xi}=60$, the network partial differential equations (39), (40) can be transformed into a system of connected differential equations. A second-order central difference scheme has been used to discretize this non-linear system of equations. The following currents (41a-41d) are implemented by means of resistors of value " $\Delta Y$ " and others currents are implemented with the voltage control current generator:

$$
\begin{aligned}
& j_{H, i, j-\Delta \eta}=\left(H_{i, j-\Delta \eta}-H_{i, j}\right) / \Delta \eta \\
& j_{H, i, j+\Delta \eta}=\left(H_{i, j}-H_{i, j+\Delta \eta}\right) / \Delta \eta \\
& j_{\mathrm{G}, \mathrm{i}, \mathrm{j}-\Delta \eta}=\left(G_{i, j-\Delta \eta}-G_{i, j}\right) / \Delta \eta \\
& j_{\mathrm{G}, \mathrm{i}, \mathrm{j}+\Delta \eta}=\left(G_{i, j}-G_{i, j+\Delta \eta}\right) / \Delta \eta
\end{aligned}
$$

A first-order central difference approximation is used for the first derivate and a second-order central difference scheme employed to discretize the non-linear system for the second derivates.

$$
\begin{aligned}
& j_{H, \eta}=\frac{\partial H}{\partial \eta} \approx \frac{H_{i, j+\Delta \eta}-H_{i, j-\Delta \eta}}{2 \Delta \eta} \\
& j_{G, \eta}=\frac{\partial G}{\partial \eta} \approx \frac{G_{i, j+\Delta \eta}-G_{i, j-\Delta \eta}}{2 \Delta \eta} \\
& j_{H, \xi}=\frac{\partial H}{\partial \xi} \approx \frac{H_{i+\Delta \xi, j}-H_{i, j}}{\Delta \xi}
\end{aligned}
$$




$$
\begin{gathered}
j_{G, \xi}=\frac{\partial G}{\partial \xi} \approx \frac{G_{i+\Delta \xi, j}-G_{i, j}}{\Delta \xi} \\
j_{f, \xi}=\frac{\partial f}{\partial \xi} \approx \frac{F_{i+\Delta \xi, j}-F_{i, j}}{\Delta \xi} \\
\frac{d j_{H, \eta}}{d \eta} \approx \frac{j_{H, i, j-\Delta \eta}-j_{H, i, j+\Delta \eta}}{\Delta \eta}=\frac{H_{i, j-\Delta \eta}+H_{i, j+\Delta \eta}-2 H_{i, j}}{\Delta \eta^{2}} \\
\frac{d j_{g, \eta}}{d \eta} \approx \frac{j_{G, i, j-\Delta \eta}-j_{G, i, j+\Delta \eta}}{\Delta \eta}=\frac{G_{i, j-\Delta \eta}+G_{i, j+\Delta \eta}-2 G_{i, j}}{\Delta \eta^{2}}
\end{gathered}
$$

In the equations (39) and (40) all the terms can be treated as a current. Therefore implementing Kirchhoff's law for electrical currents from circuit theory, the network model is generated. To introduce the boundary conditions, voltage sources are employed to simulate constant values of velocity and temperature. Similarly, the equations (31) and (32) (which correspond to Case II) can be modeled by means of NSM and it is possible to obtain the numerical solutions to $\xi=0$. These are subsequently input as boundary conditions for a solution for $\xi>0$, which is twodimensional.

\section{COMPUTATIONS AND DISCUSSION}

The heat transfer regime is controlled by six parameters- $H a_{x}, \varepsilon, n, G r_{x}, P r$, $\xi$. In most cases we have plotted $C_{f x} G r_{x}{ }^{1 / 4}=\frac{\partial^{2} F(\xi, 0]}{\partial \eta^{2}}$ versus $\xi$ and $N u_{x} G r_{x}{ }^{-1 / 4}=-\frac{\partial G(\xi, 0)}{\partial \eta}$ versus $\xi$. The range of the abscissa is taken as $0 \leq \xi \leq 5$. Default values are set as $H a_{x}=1, \varepsilon=0, n=0.4, G r_{x}=10$, $\operatorname{Pr}=0.72$. In this study as in Alam et al [28] only suction is considered i.e. $\xi>0$. To validate the present NSM solutions we have made comparisons with the classical study of Hering and Grosh [7] and also more recent implicit finite difference Keller-box solutions presented by Hossain et al [14]. These are presented in Tables 1 and 2. Excellent correlation has been achieved with both [7] and [14] over several $n$ values and also a wide range of $\operatorname{Pr}$ values, testifying to the validity of the NSM technique. 


\begin{tabular}{|l|l|l|l|l|}
\hline$n \rightarrow$ & \multicolumn{2}{|c|}{0.0} & \multicolumn{2}{c|}{1.0} \\
\hline Method & $\frac{\partial^{2} F(\xi, 0]}{\partial \eta^{2}}$ & $-\frac{\partial G(\xi, 0]}{\partial \eta}$ & $\frac{\partial^{2} F(\xi, 0]}{\partial \eta^{2}}$ & $-\frac{\partial G(\xi, 0]}{\partial \eta}$ \\
\hline $\begin{array}{l}\text { Hering \& } \\
\text { Grosh [7] }\end{array}$ & 0.81959 & 0.45120 & 0.72480 & 0.56699 \\
\hline $\begin{array}{l}\text { Hossain et } \\
\text { al. [14] }\end{array}$ & 0.81894 & 0.45110 & 0.72362 & 0.56659 \\
\hline $\begin{array}{l}\text { Present } \\
\text { NSM }\end{array}$ & 0.81928 & 0.45310 & 0.72462 & 0.56871 \\
\hline
\end{tabular}

Table 1; Comparison for non-conducting case $\left[\mathrm{Ha}_{x}=0\right.$ ], for $\xi=0, n=0$ and 1.0, $\varepsilon=0$ and $\mathrm{Pr}=$ 0.7

\begin{tabular}{|l|l|l|l|l|l|l|}
\hline \multirow{2}{*}{$\begin{array}{l}\text { Pr } \\
\text { Method }\end{array}$} & $\frac{\partial^{2} F(\xi, 0]}{\partial \eta^{2}}$ & $-\frac{\partial G(\xi, 0]}{\partial \eta}$ & $\frac{\partial^{2} F(\xi, 0]}{\partial \eta^{2}}$ & $-\frac{\partial G(\xi, 0]}{\partial \eta}$ & $\frac{\partial^{2} F(\xi, 0]}{\partial \eta^{2}}$ & $-\frac{\partial G(\xi, 0]}{\partial \eta}$ \\
\hline $\begin{array}{l}\text { Hossain et al. [14] } \\
(\xi=0)\end{array}$ & 1.23231 & 0.08828 & 1.09069 & 0.18300 & 1.01332 & 0.24584 \\
\hline $\begin{array}{l}\text { Present NSM } \\
(\xi=0)\end{array}$ & 1.23550 & 0.08851 & 1.09590 & 0.18346 & 1.00939 & 0.24722 \\
\hline $\begin{array}{l}\text { Hossain et al. [14] } \\
(\xi=0.4)\end{array}$ & 1.48846 & 0.09021 & 1.25262 & 0.19304 & 1.14437 & 0.26630 \\
\hline $\begin{array}{l}\text { Present NSM } \\
(\xi=0.4)\end{array}$ & 1.48345 & 0.09025 & 1.25257 & 0.19323 & 1.14566 & 0.26645 \\
\hline $\begin{array}{l}\text { Hossain et al. [14] } \\
(\xi=1.0)\end{array}$ & 1.78637 & 0.09297 & 1.49247 & 0.20726 & 1.32826 & 0.29731 \\
\hline $\begin{array}{l}\text { Present NSM } \\
(\xi=1.0)\end{array}$ & 1.78679 & 0.09290 & 1.49287 & 0.20719 & 1.32856 & 0.29712 \\
\hline $\begin{array}{l}\text { Hossain et al. [14] } \\
(\xi=4.0)\end{array}$ & 3.37732 & 0.10585 & 2.26468 & 0.28720 & 1.66712 & 0.47828 \\
\hline $\begin{array}{l}\text { Present NSM } \\
(\xi=4.0)\end{array}$ & 3.37712 & 0.10574 & 2.26455 & 0.28713 & 1.66735 & 0.47849 \\
\hline
\end{tabular}

Table 2; Comparison for non-conducting case $\left[\mathrm{Ha}_{x}=0\right]$, for $\xi=0,0.4,1.0$ and 4.0 with $n=$ $0.5, \varepsilon=0$ and $\operatorname{Pr}=0.01,0.05$ and 0.1 
Figures 2 and 3 show the influence of the Prandtl number, $P r$, on $C_{f x} G r_{x}{ }^{1 / 4}=\frac{\partial^{2} F(\xi, 0]}{\partial \eta^{2}}$ and $N u_{x} / G r_{x}^{1 / 4}=-\frac{\partial G(\xi, 0)}{\partial \eta}$. An increase in $\operatorname{Pr}$ strongly reduces the skin friction at the cone surface $(\xi=0)$, a trend sustained along the cone slant surface i.e. with increasing $\xi$. For $\operatorname{Pr}=3,5$ and 7, the skin friction becomes negative far from the cone apex (i.e. leading edge of the boundary layer) indicating back flow. For higher $\operatorname{Pr}$ fluids the thermal diffusivity is reduced and momentum diffusivity is increased i.e. momentum is diffused faster than heat. Increasing Prandtl number implies an increase in dynamic viscosity and a decrease in thermal conductivity of the fluid. As such lower Prandtl numbers correspond to gases (low viscosity and high thermal conductivity) and higher Prandtl numbers to oils (high viscosity, lower thermal conductivities). For low Prandtl number the fluid will flow faster i.e. velocities will be increased which will enhance skin friction at the cone surface, as testified by figure 2 . Conversely with an increase in $\mathrm{Pr}$, local Nusselt number function, $N u_{x} G r_{x}^{-1 / 4}$, is significantly increased. The response in figure 3 is also much more gradual and approximately linear compared with the skin friction (figure 2). Clearly an increase in $\mathrm{Pr}$ implies that thermal diffusivity exceeds momentum diffusivity so that heat is diffused at a faster rate than momentum, leading to an escalation in heat transfer gradient at the cone surface. $N u_{x} G r_{x}^{-1 / 4}$ is maximized at the trailing edge (maximum $\xi$ ) and minimized at the leading edge (apex i.e. $\xi=0$ ) for any value of Prandtl number.

Figures 4 and 5 depict the response of $C_{f x} G r_{x}{ }^{1 / 4}=\frac{\partial^{2} F(\xi, 0]}{\partial \eta^{2}}$ and $N u_{x} / G r_{x}{ }^{1 / 4}=-\frac{\partial G(\xi, 0)}{\partial \eta}$ to local Hartmann number, $H a_{x}$, with coordinate along the cone surface, $\xi$. In the momentum eqn (24), the hydromagnetic term incorporating $H a_{x}, \frac{H a_{x}{ }^{2}}{G r_{x}{ }^{1 / 2}} \frac{\partial F}{\partial \eta}$ is a drag force term. Increasing $H a_{x}$, with constant $G r_{x}$, will therefore serve to decelerate the flow in the momentum boundary layer. We note that this parameter physically can be regarded as the ratio of magnetic viscous force to hydrodynamic viscous force. Cramer and Pai [38] have shown that magnetism has a strong effect on velocity profiles, causing a reduction in flow and shear stresses. In accordance with this, we observe in figure 4 that as $H a_{x}$ is increased from 0 (non-conducting case) through $0.5,1.0,2.0$ to 5.0 , a strong decrease is induced in the skin friction. Skin friction is also found to be diminished with increasing distance along the cone surface from the leading edge (apex). This behaviour is consistent with that reported in numerous other hydromagnetic cone flow studies including Sluyter and Touryan [21], Surma Devi et al [22] and also exhibits the correct physical response 
for magnetohydrodynamic boundary layers as described by Cramer and Pai [38]. In figure 5 $N u_{x} G r_{x}^{-1 / 4}$, is seen to be marginally decreased with increasing $H a_{x}$ values, mainly in the vicinity of the leading edge. Increasing magnetic field will increase the Lorentzian drag force, $\frac{H a_{x}{ }^{2}}{G r_{x}{ }^{1 / 2}} \frac{\partial F}{\partial \eta}$, which causes the fluid to do supplementary work. This is manifested by an increase in thermal energy in the fluid which heats the flow and decreases heat transfer rates at the cone surface. We further note that with increasing distance from the leading edge, the $N u_{x} G r_{x}^{-1 / 4}$, profiles all converge, a pattern sustained to the trailing edge. This indicates that magnetic field exerts no tangible effect after a certain separation from the apex of the cone. A similar response has been identified by for example Singh et al [23] and for flat plate hydromagnetic convection, by Cramer and Pai [38]. Heat transfer gradient is therefore maximized for the non-conducting case and minimized for the strong magnetic field case. The latter is of immense potential therefore in magnetic field control of for example spacecraft re-entry surface heat transfer rates, in the vicinity of the nose. The electrically-conducting nature of plasma-type flows in re-entry problems and rarified gases in high altitude hypersonic heat transfer can therefore also be exploited to advantage with magnetic field control technology.

Figures 6 and 7 illustrate the distribution of $C_{f x} G r_{x}{ }^{1 / 4}=\frac{\partial^{2} F(\xi, 0]}{\partial \eta^{2}} \quad$ and $N u_{x} / G r_{x}{ }^{1 / 4}=-\frac{\partial G(\xi, 0)}{\partial \eta}$ with various temperature power law index values $(n)$ with streamwise coordinate, $\xi$. This parameter arises frequently in both the momentum and energy boundary layer equations (24) and (25). The case, $n=0$ implies isothermal flow. For $n>0$, the regime is nonisothermal. With increasing $n$ a steeper temperature gradient arises at the cone surface. As a result skin friction (figure 6) is found to be strongly reduced at $(\xi=0)$ and in close proximity $(0<$ $\xi<2)$ to the cone apex. With further distance along the cone surface, all profiles are found to merge such that no significant effect of $n$ on skin friction is experienced for $\xi>3$.

Conversely, as expected, $N u_{x} G r_{x}^{-1 / 4}$ values (figure 7) are enhanced at the leading edge $(\xi=0)$ with an increase in $n$. Maximum local Nusselt number function and therefore heat transfer rate arises for the strongly non-isothermal case $(n=0.9)$ and the minimum for the isothermal case $(n=$ 0 ). All profiles converge at $\xi \sim 2$, demonstrating very little response to $n$ with further progress along the cone slant surface. 


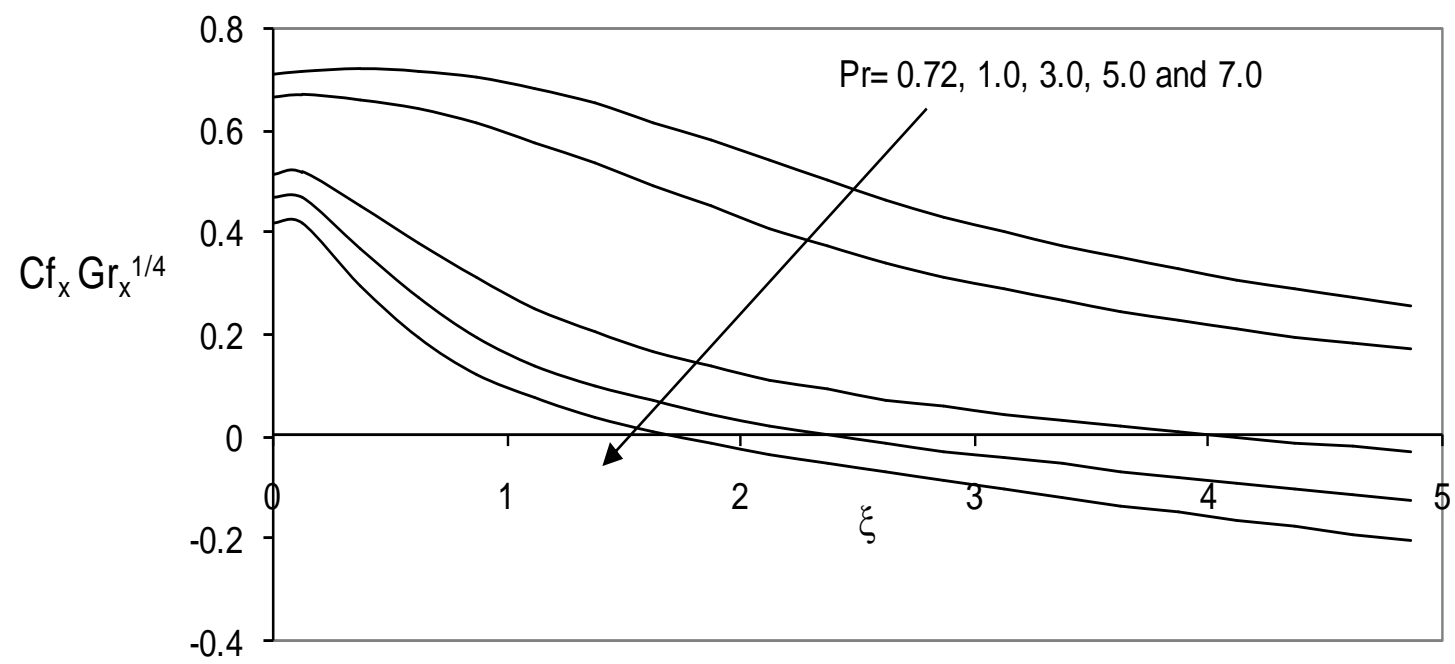

Figure 2: Skin friction versus streamwise coordinate for various Prandtl numbers $(\mathrm{Pr})$

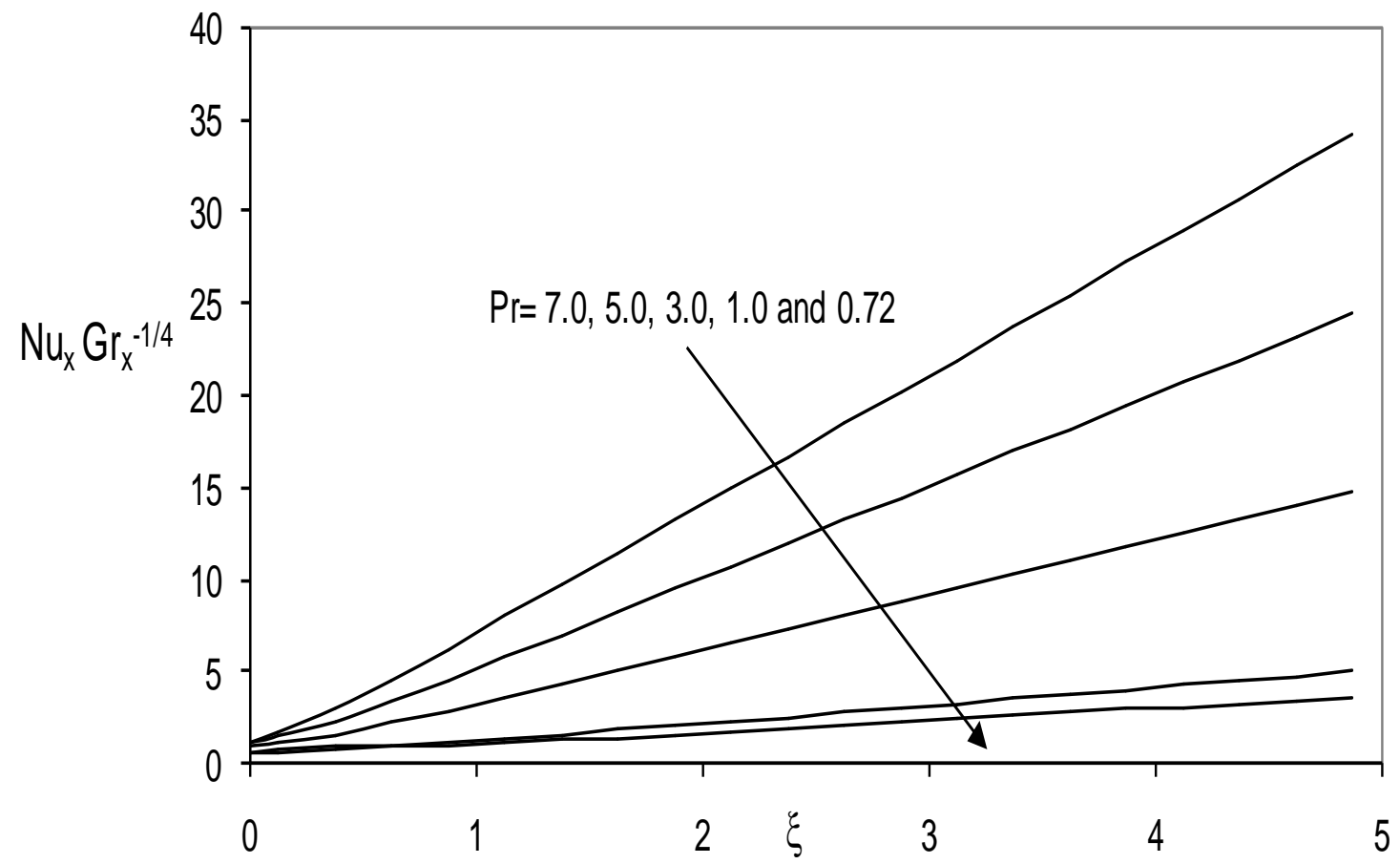

Figure 3: Nusselt number function versus streamwise coordinate for various Prandtl numbers $(P r)$ 


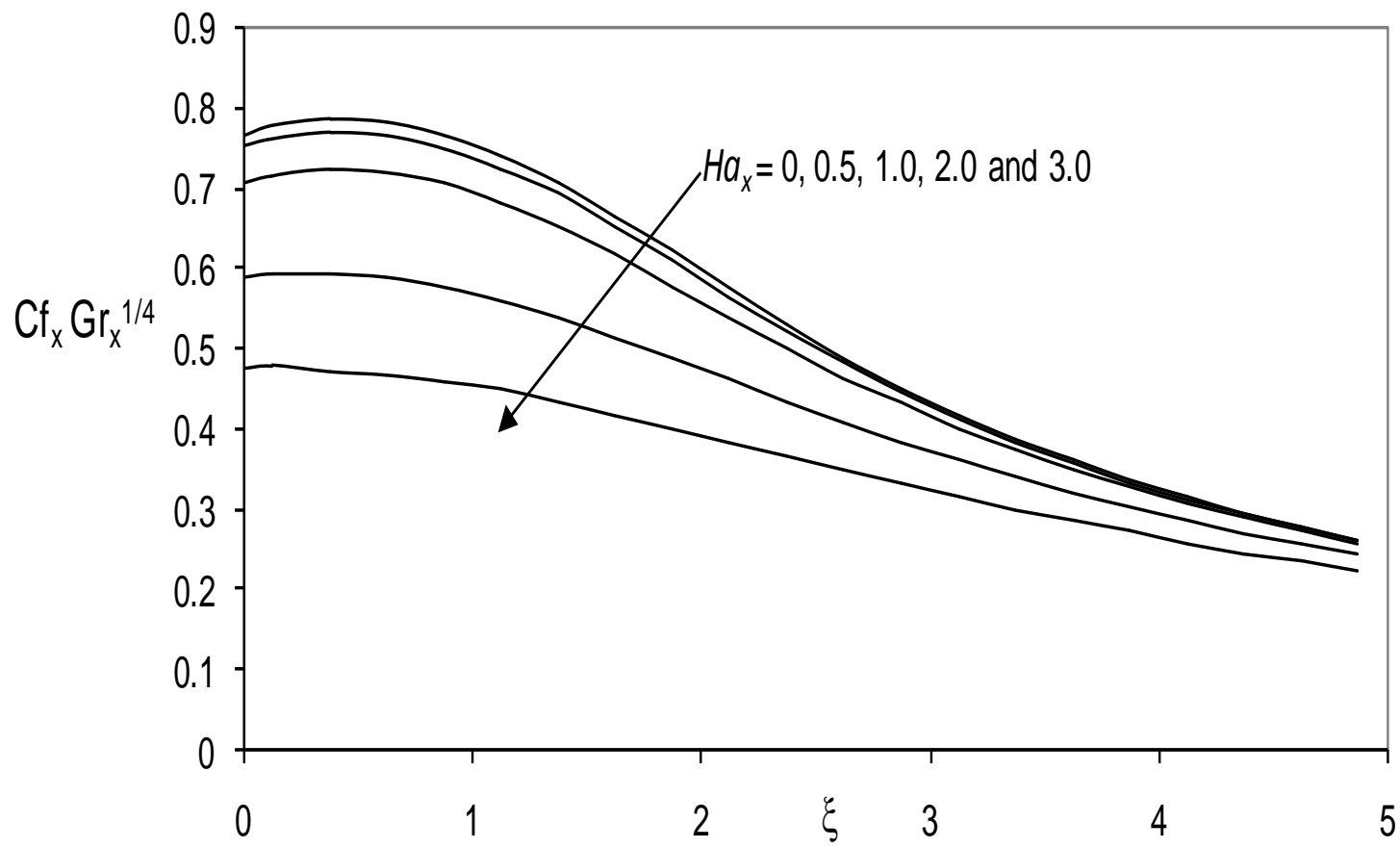

Figure 4 : Skin friction versus streamwise coordinate for various local Hartmann numbers $\left(H a_{x}\right)$

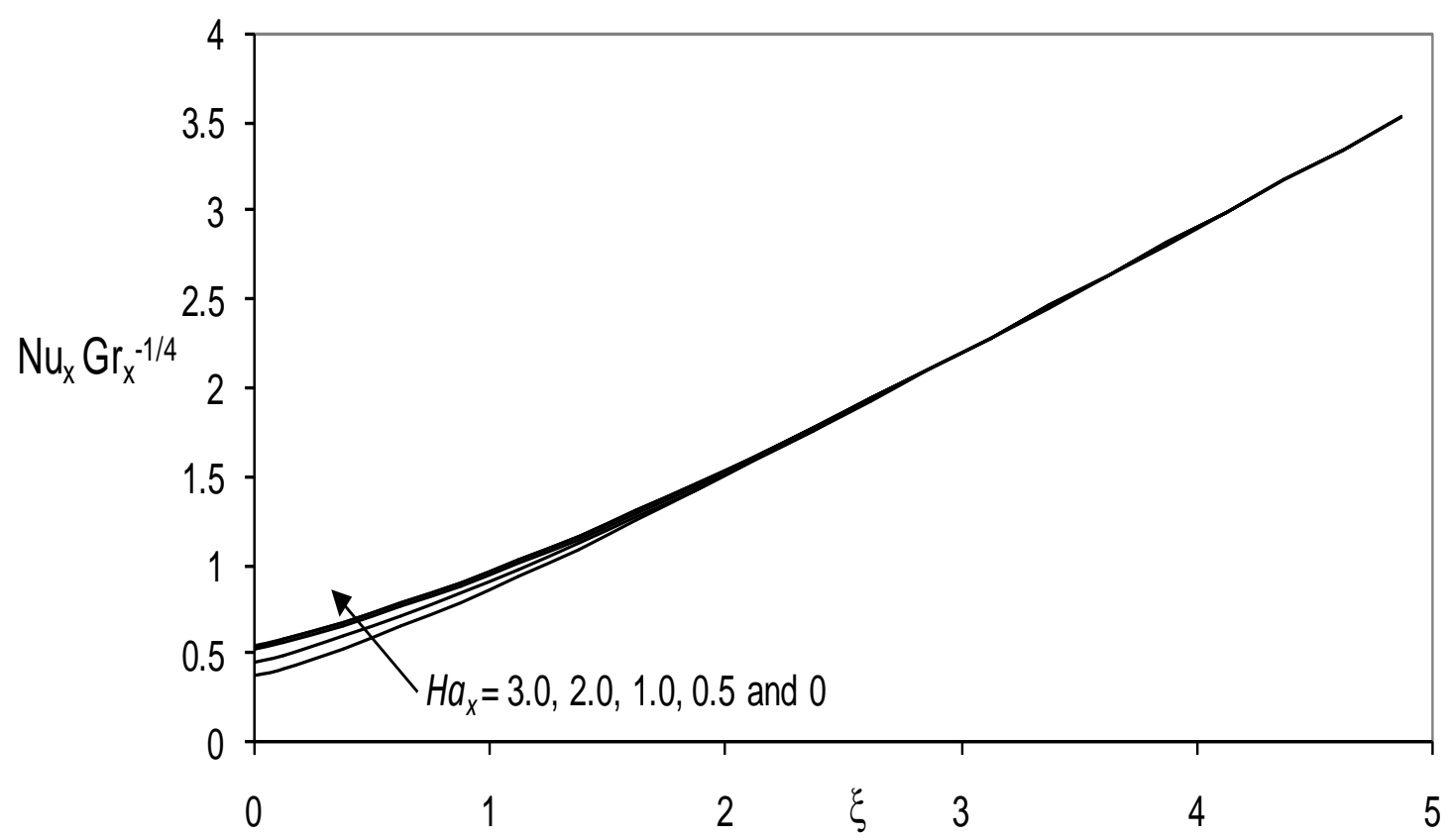

Figure 5 : Nusselt number function versus streamwise coordinate for various local Hartmann numbers $\left(H a_{x}\right)$ 


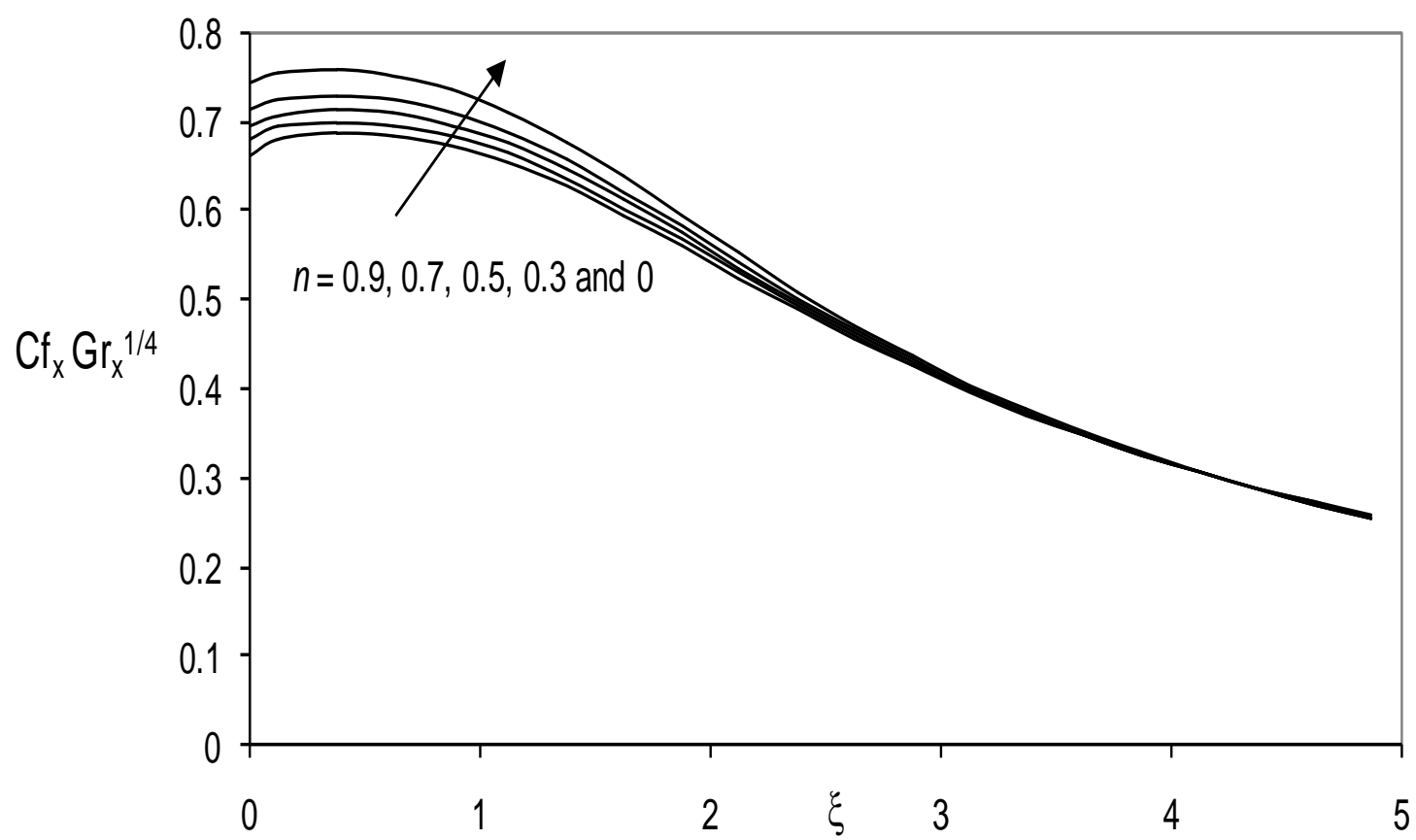

Figure 6 : Skin friction versus streamwise coordinate for various temperature power law index values $(n)$

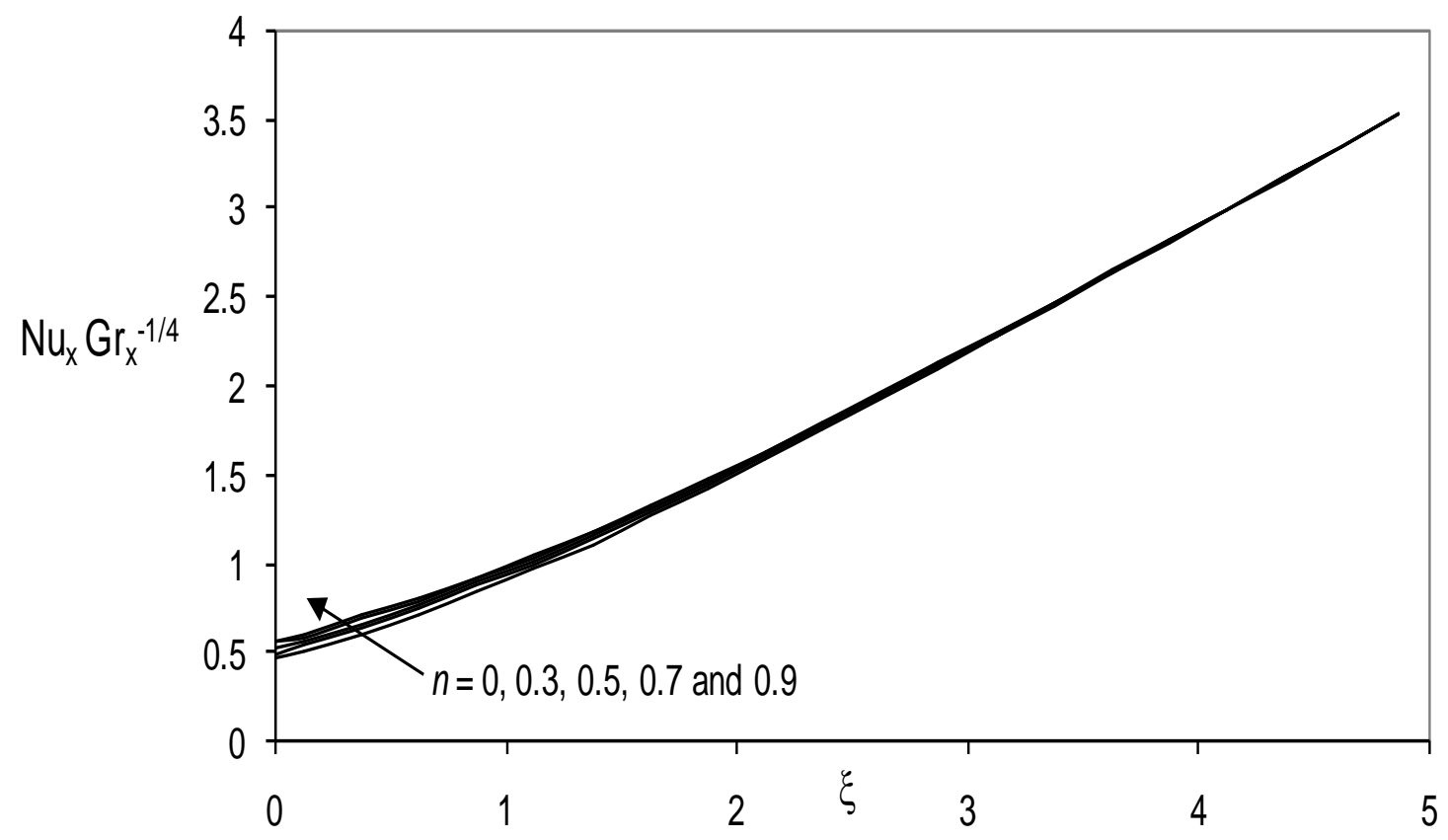

Figure 7 : Local Nusselt number function versus streamwise coordinate for various temperature power law index values $(n)$ 


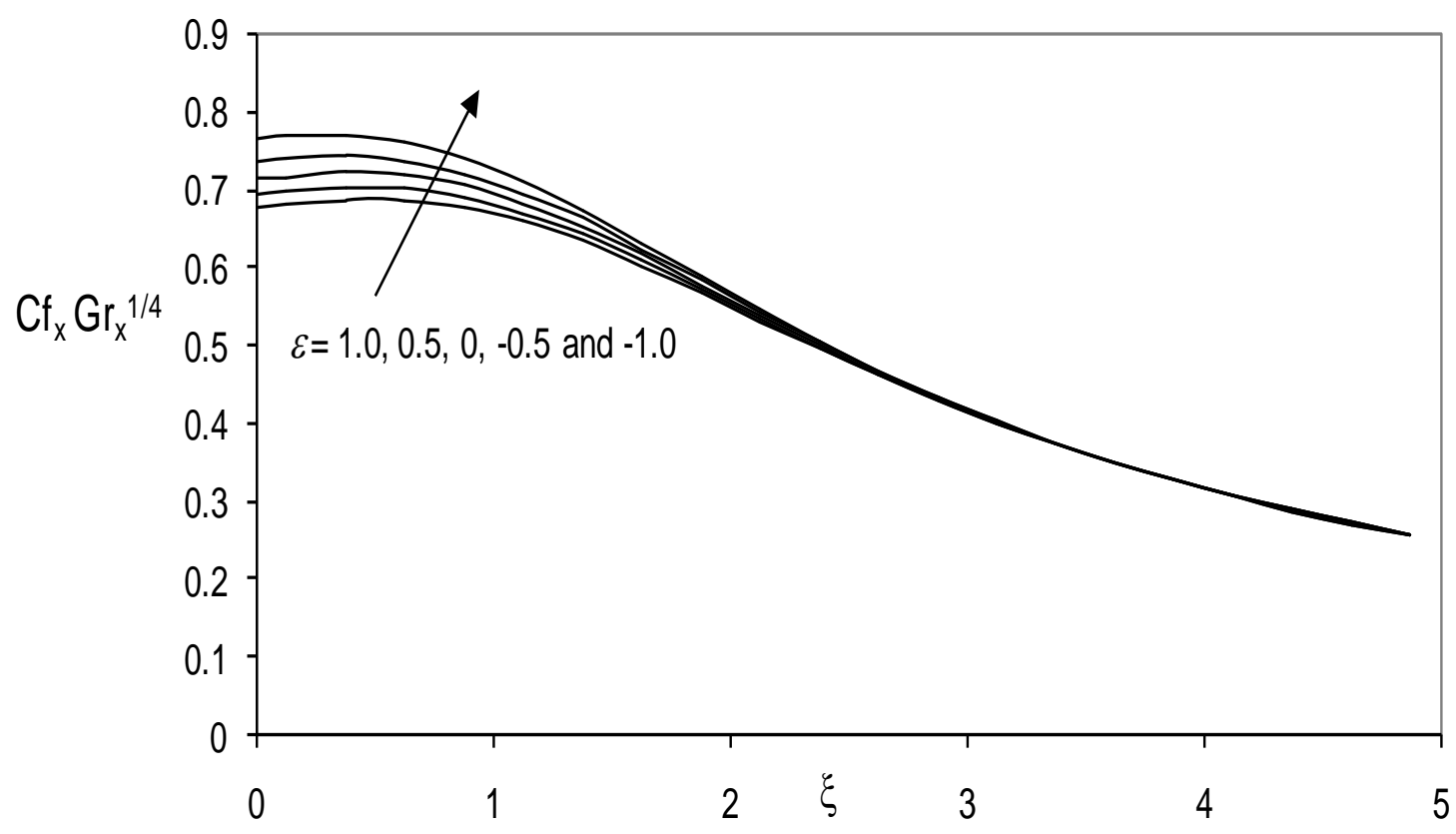

Figure 8 : Skin friction versus streamwise coordinate for various pressure work parameters $(\varepsilon)$

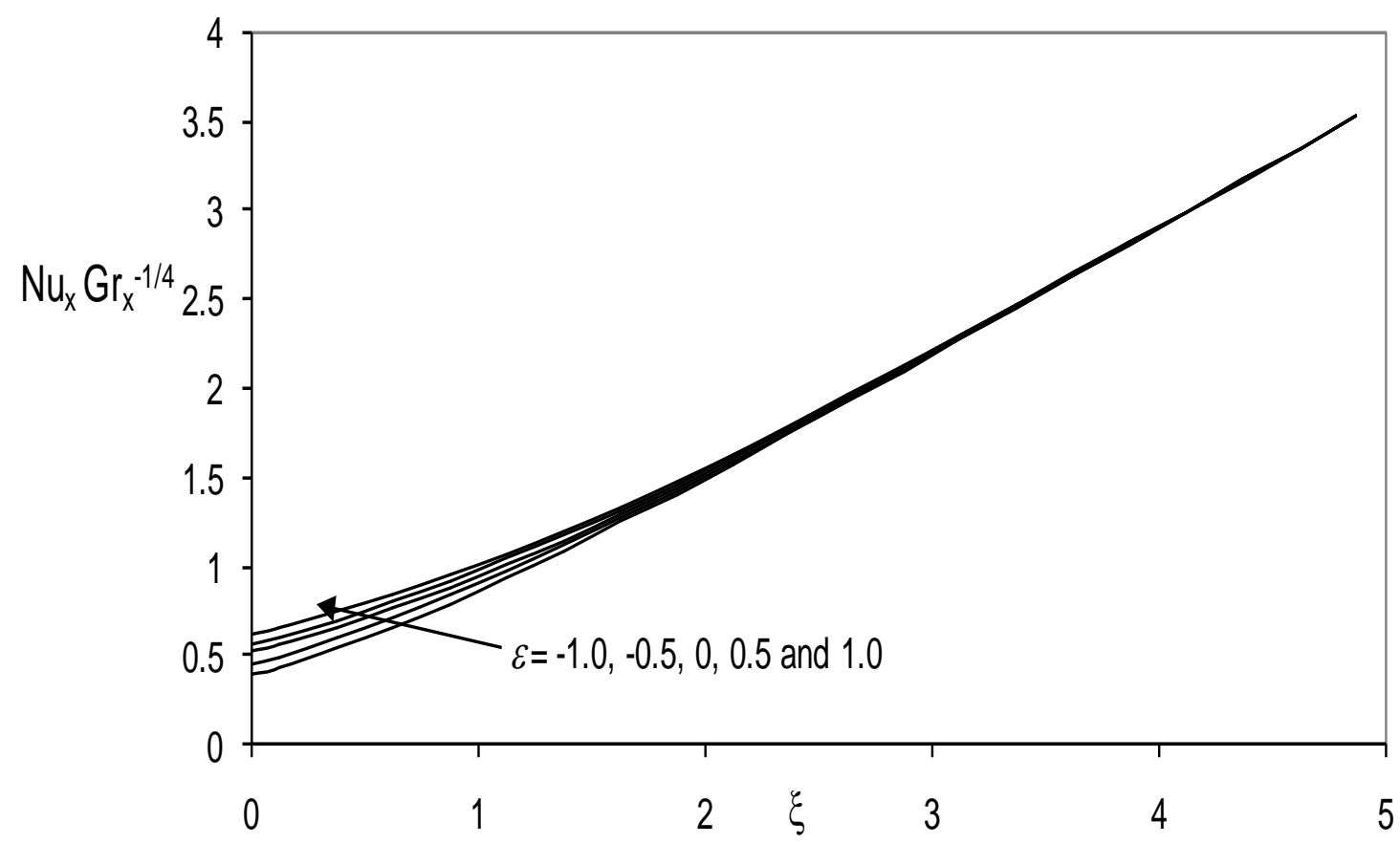

Figure 9 : Local Nusselt number versus streamwise coordinate for various pressure work parameters $(\varepsilon)$ 


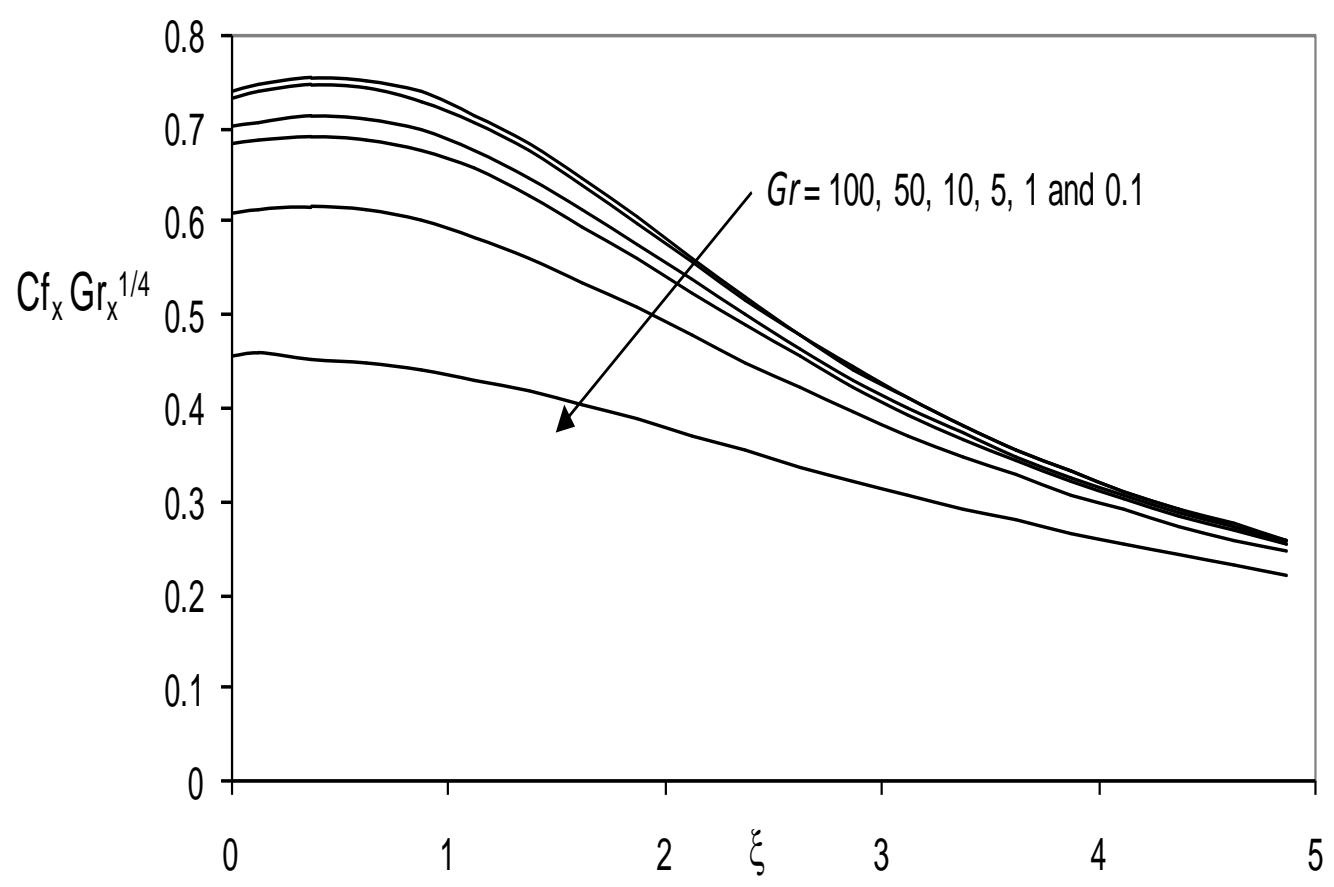

Figure 10 : Skin friction versus streamwise coordinate for various Grashof numbers $(G r)$

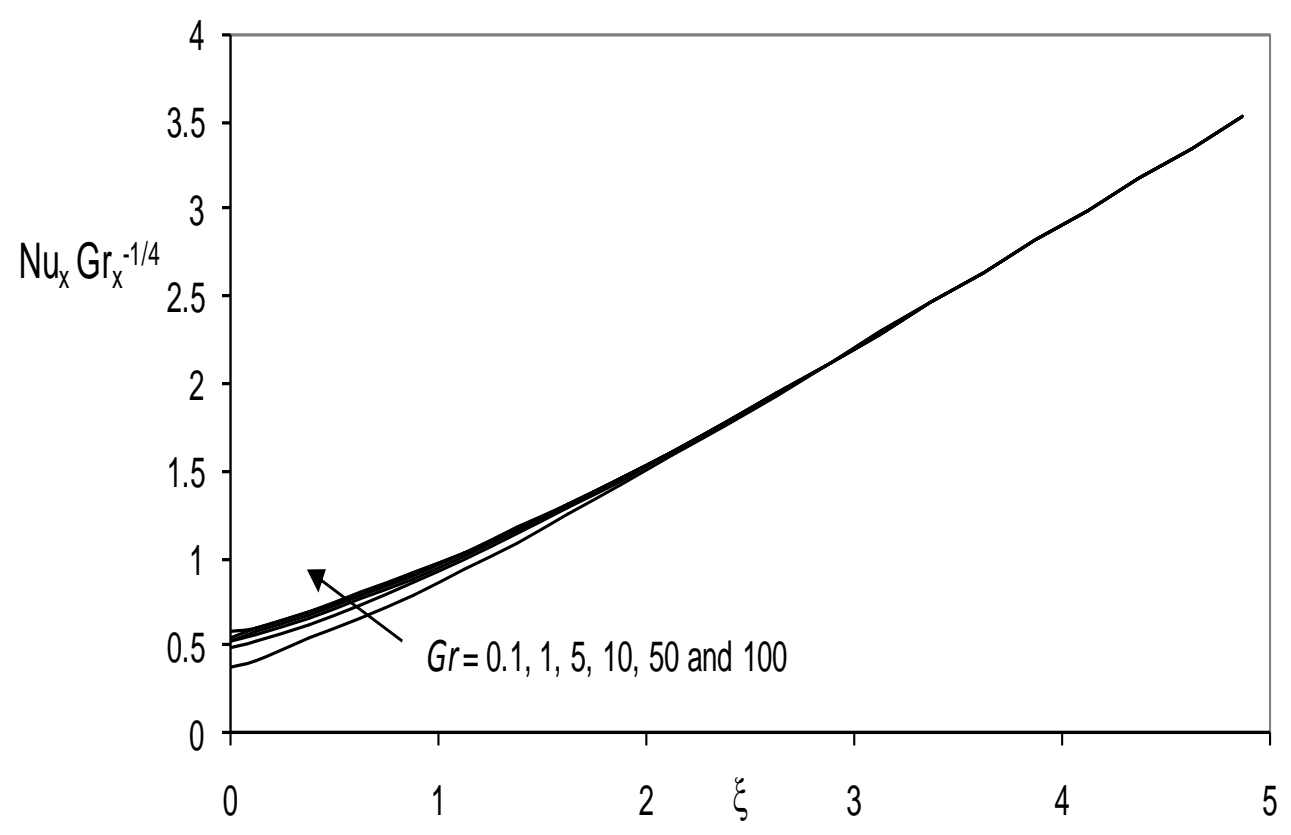

Figure 11 : Local Nusselt number function versus streamwise coordinate for various Grashof numbers $(G r)$ 


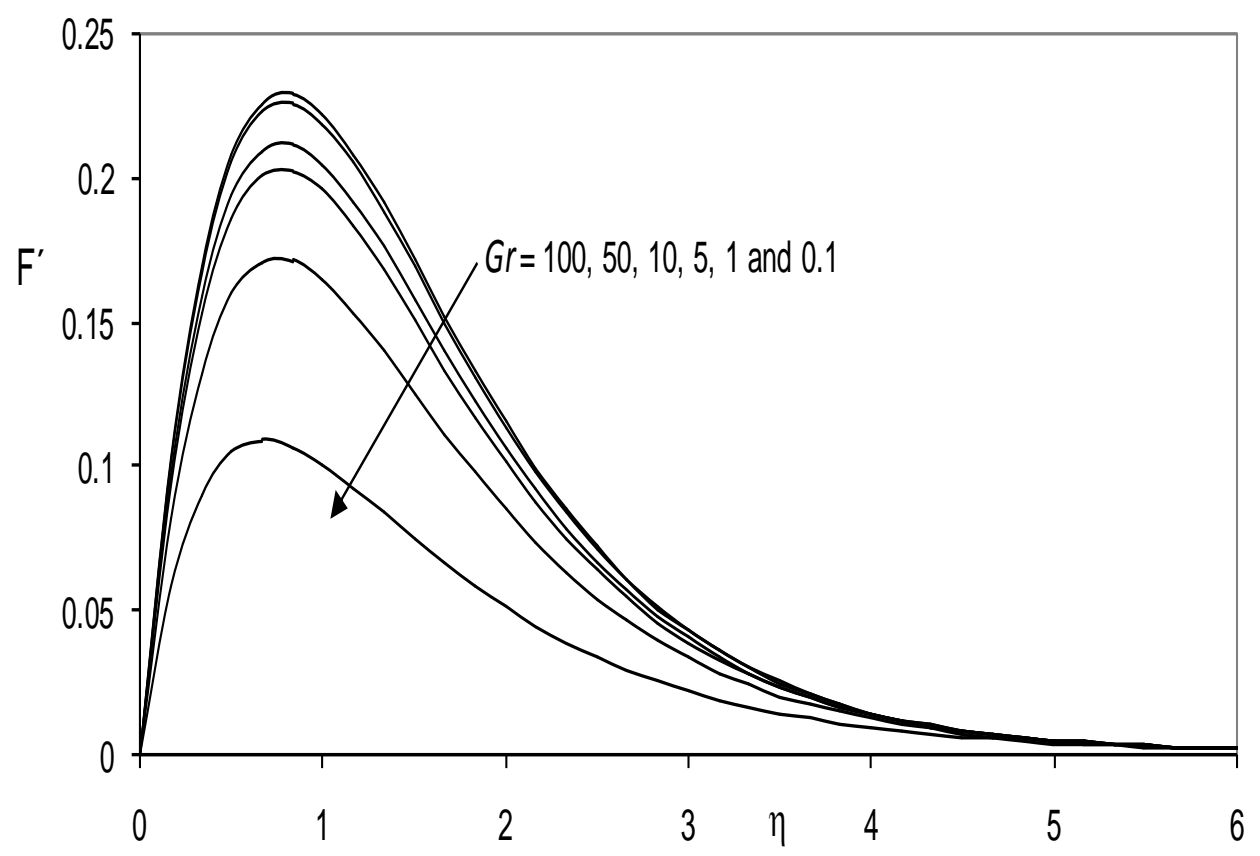

Figure 12 : Velocity versus spanwise coordinate for various Grashof numbers $(\mathrm{Gr})$

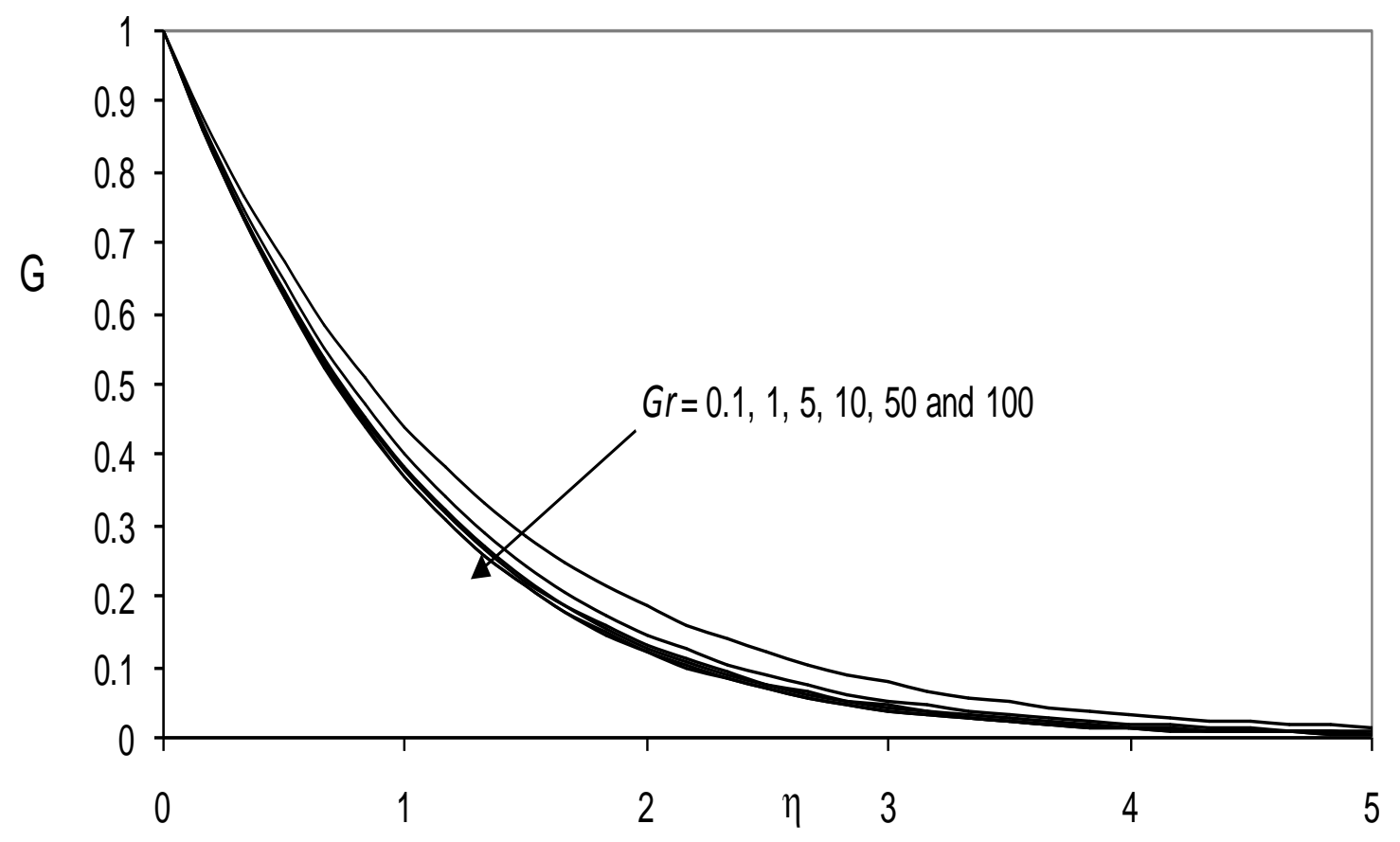

Figure 13 : Temperature versus spanwise coordinate for various Grashof numbers $(G r)$ 


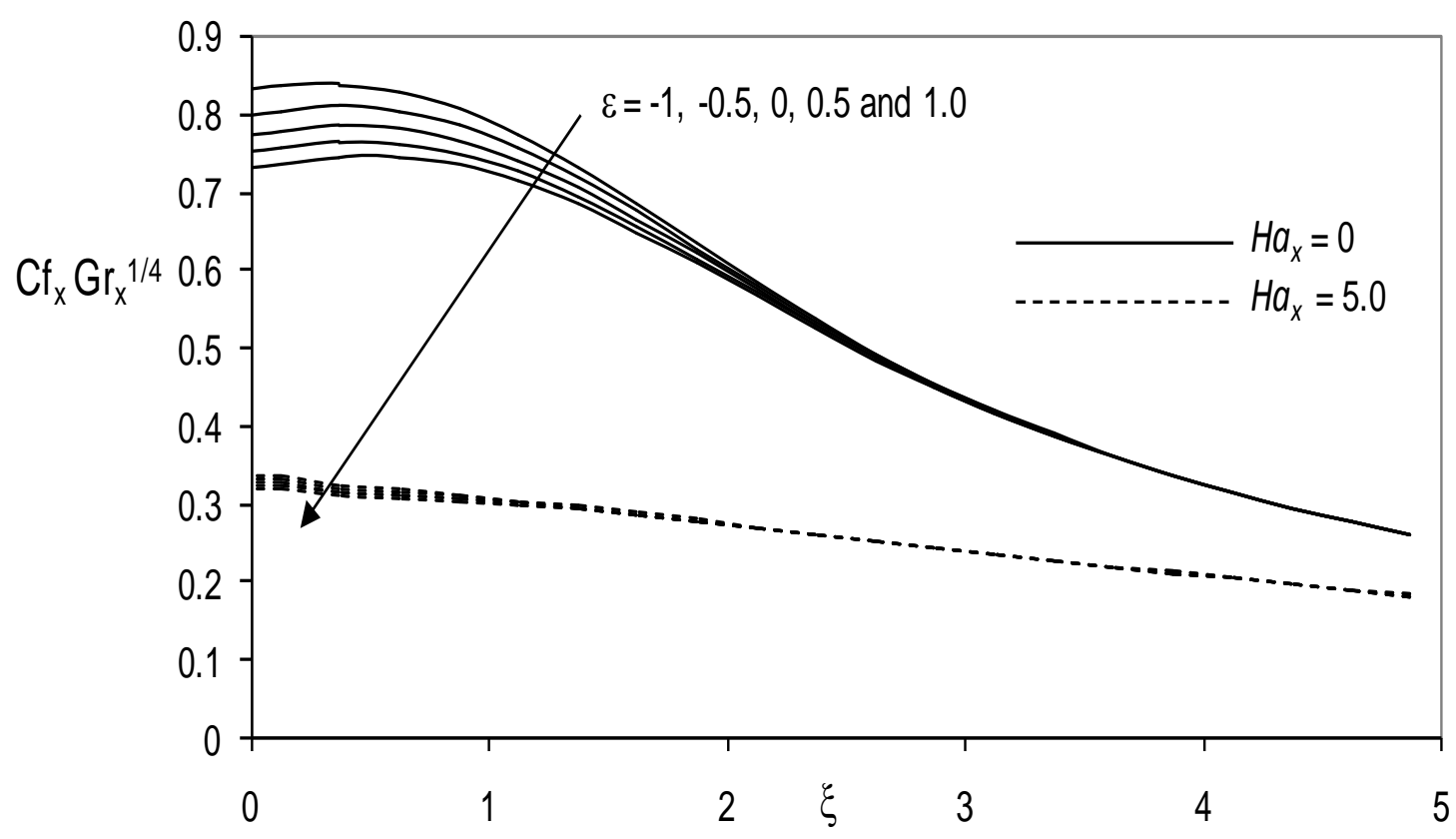

Figure 14 : Skin friction versus streamwise coordinate for collective effects of various local Hartmann numbers $\left(H a_{x}\right)$ and pressure work parameters $(\varepsilon)$

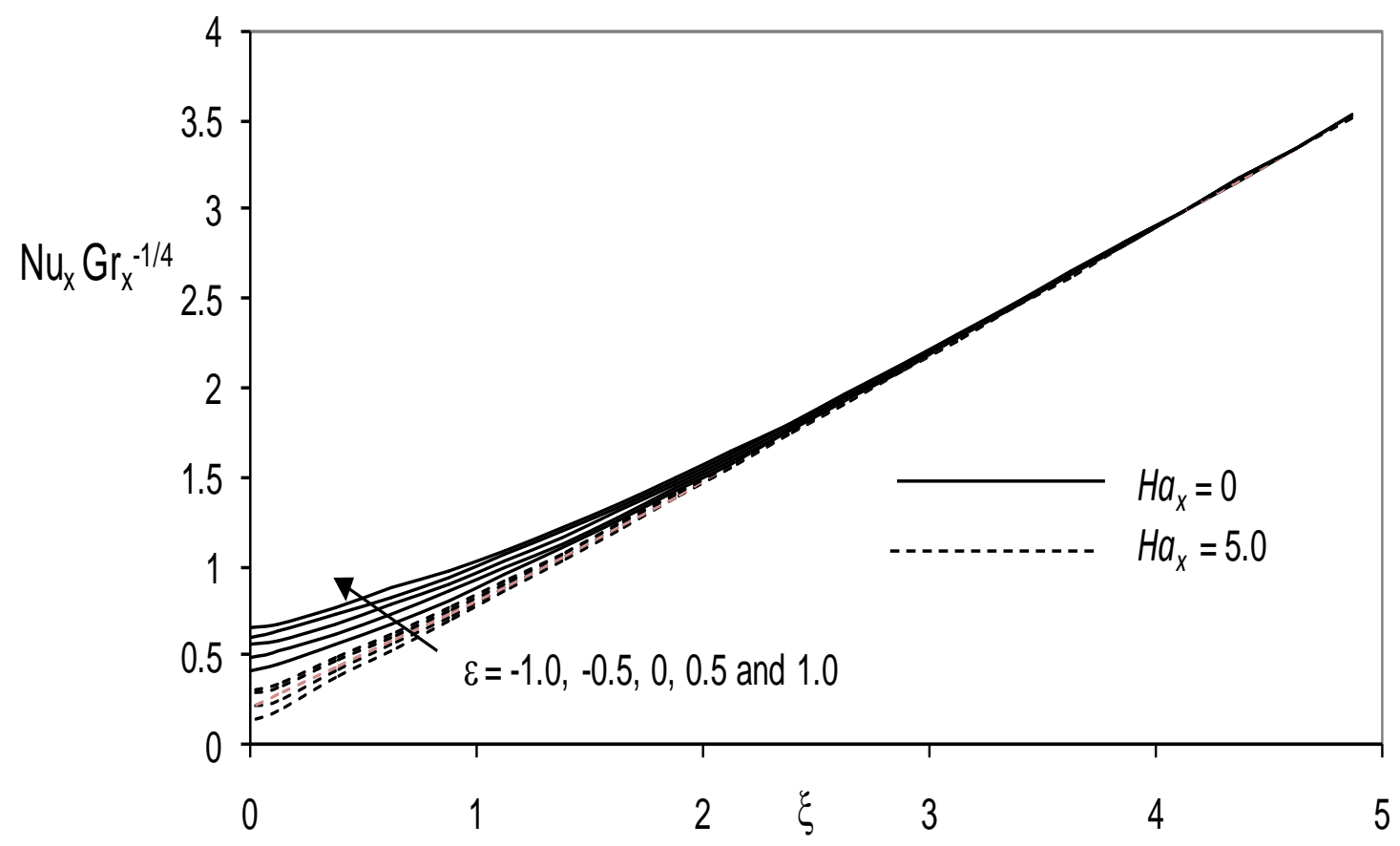

Figure 15 : Local Nusselt number function versus streamwise coordinate for collective effects of various local Hartmann numbers $\left(H a_{x}\right)$ and pressure work parameters $(\varepsilon)$ 


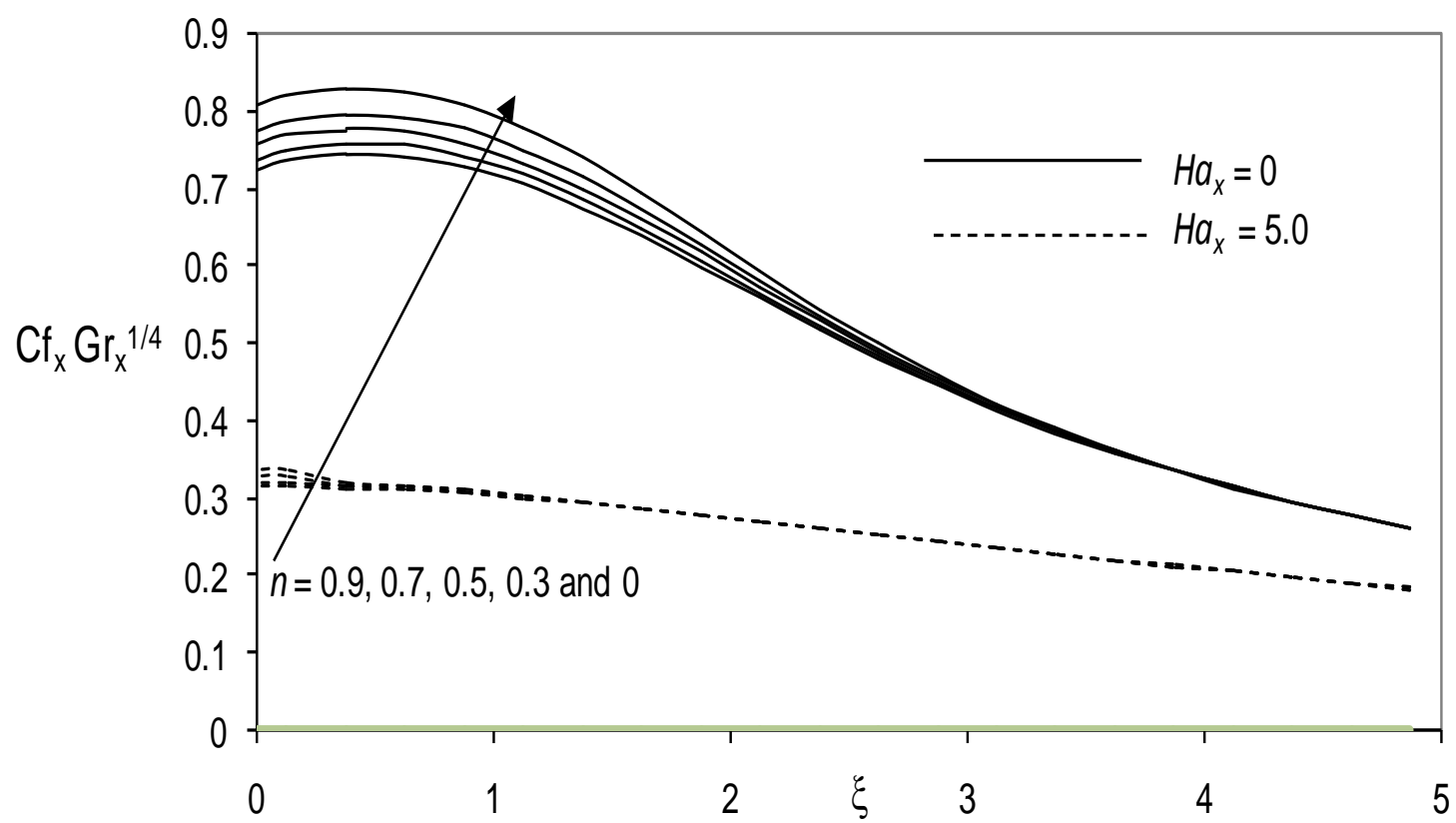

Figure 16: Skin friction versus streamwise coordinate for collective effects of various local Hartmann numbers $\left(H a_{x}\right)$ and temperature power law index parameters $(n)$

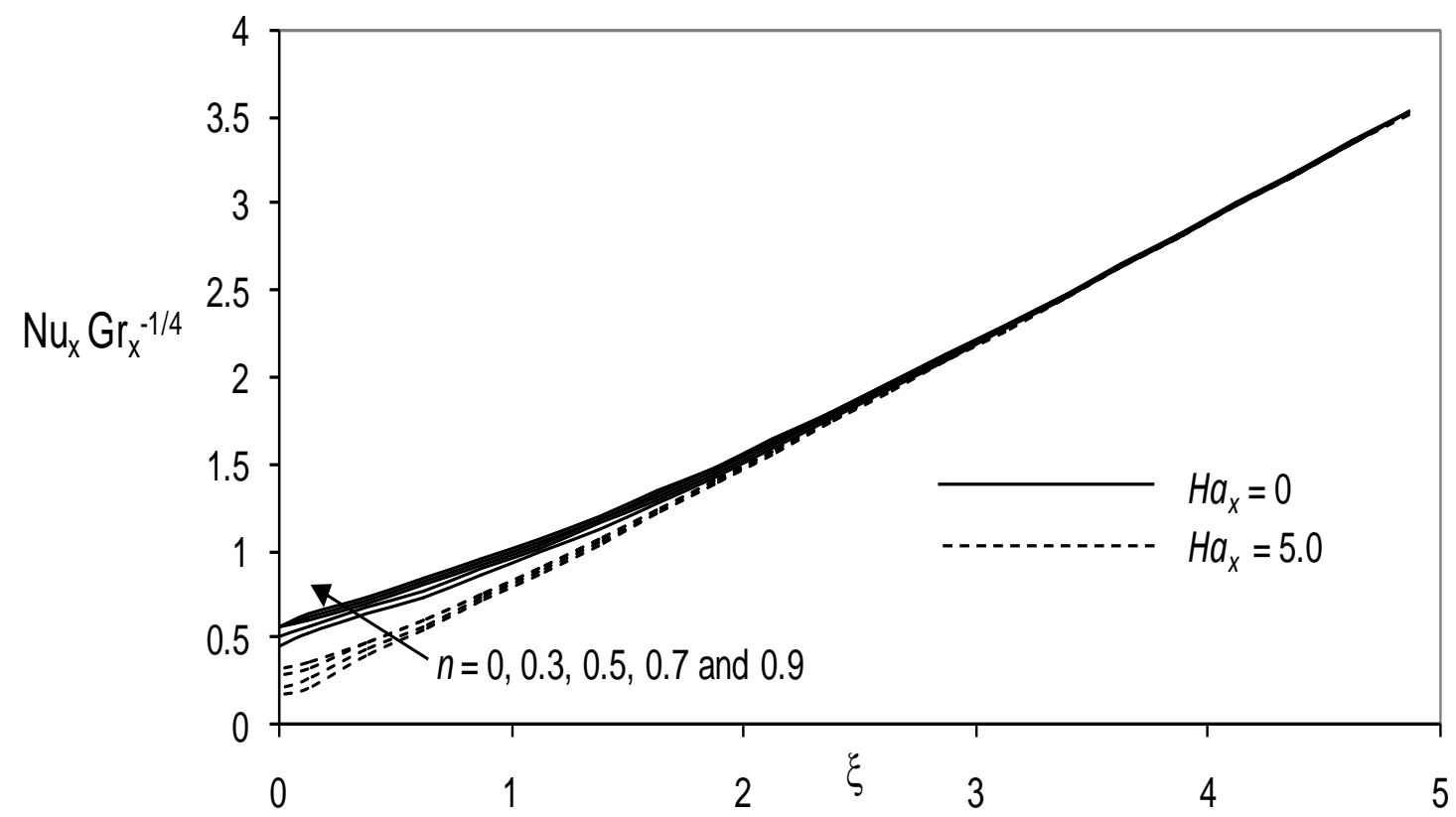

Figure 17: Local Nusselt number function versus streamwise coordinate for collective effects of various local Hartmann numbers $\left(H a_{x}\right)$ and temperature power law index parameters $(n)$ 


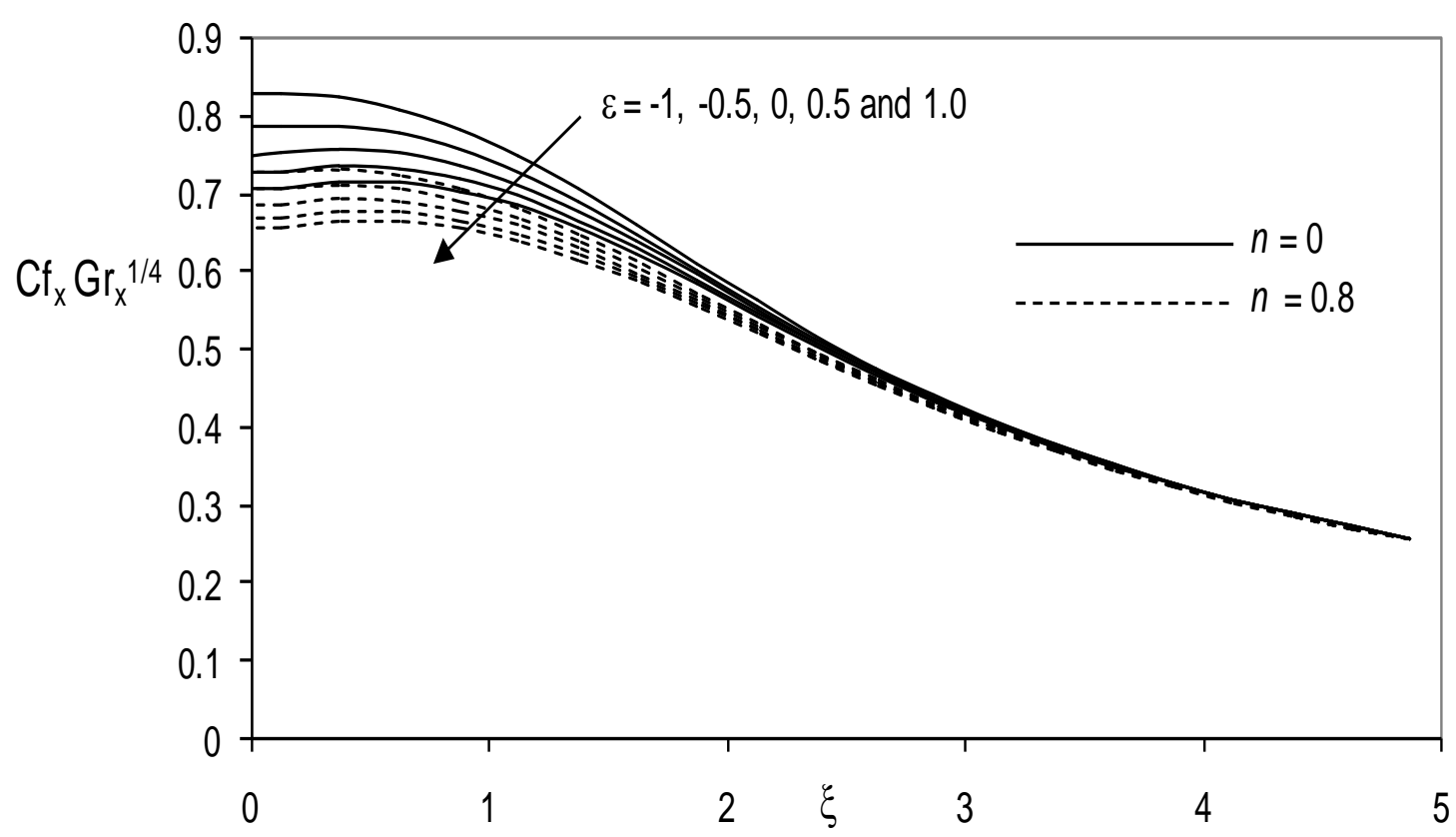

Figure 18 : Skin friction versus streamwise coordinate for collective effects of various pressure work parameters $(\varepsilon)$ and temperature power law index parameters $(n)$

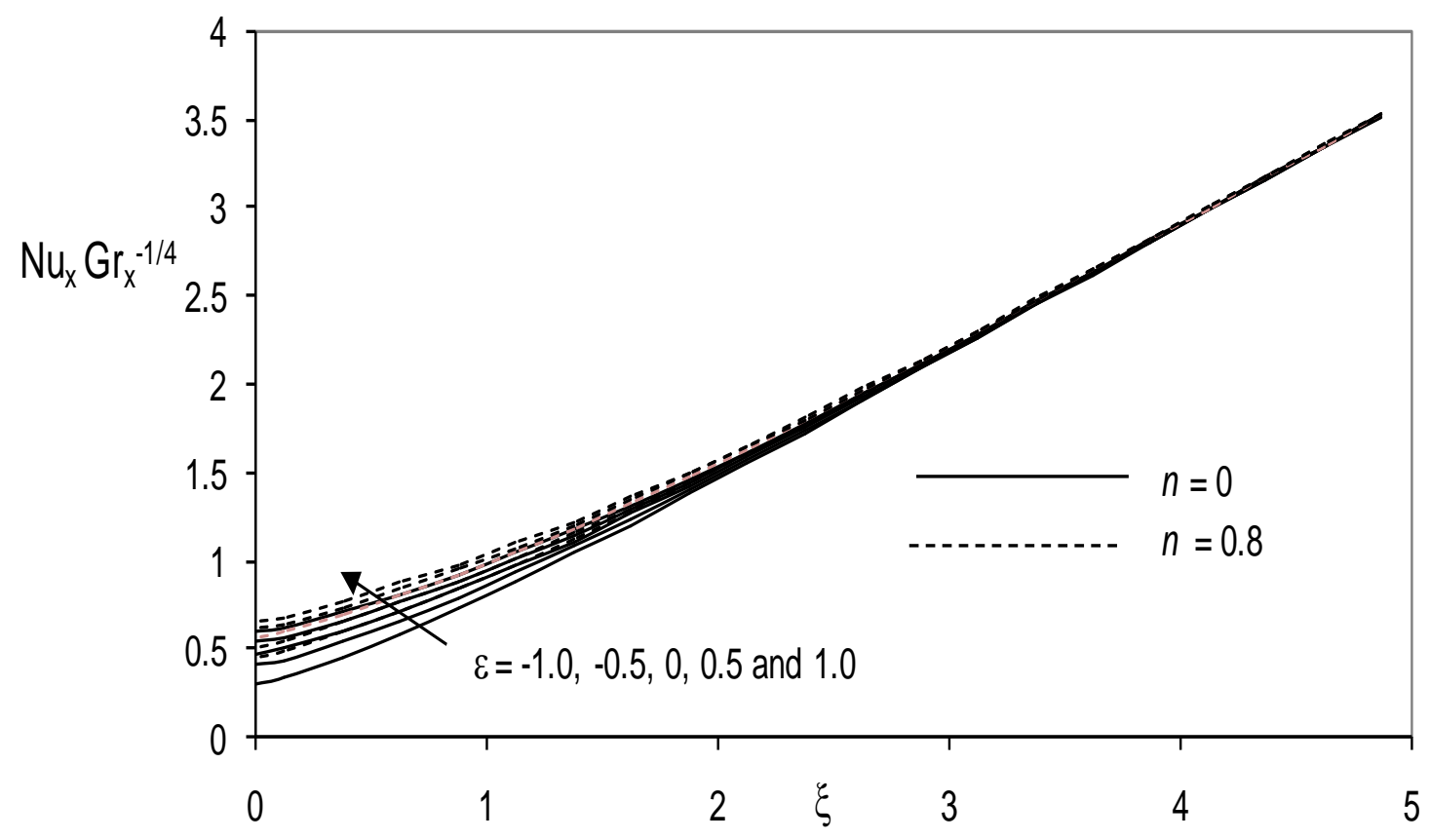

Figure 19: Local Nusselt number versus streamwise coordinate for collective effects of various pressure work parameters $(\varepsilon)$ and temperature power law index parameters $(n)$ 
Figures 8 and 9 illustrate the effect of pressure work parameters $(\varepsilon)$ on $C_{f x} G r_{x}^{1 / 4}$ and $N u_{x} G r_{x}^{=1 / 4}$. Skin friction clearly decreases with a positive increase in $\varepsilon$ and increases with a negative increase in $\varepsilon$. The parameter $\varepsilon=\frac{g \beta x}{c_{p}}$ will aid the momentum boundary layer flow when negative, since pressure stress work will not dissipate energy of the flow. Conversely with positive $\varepsilon$ the pressure stress effect will expend energy. For $\varepsilon=0$ pressure (stress) work effects are neglected in the boundary layer physics. As a result the flow will be accelerated with negative $\varepsilon$ and decelerated with positive $\varepsilon$, which physically accounts for skin friction response shown in figure 8. The $\varepsilon$ parameter was also used by Joshi and Gebhart [40] and our results agree with their general findings. Ackroyd earlier [41] also studied pressure stress work effects although for the much simpler case of flat plate convection, showing that pressure work effects are generally greater than viscous dissipation effects. Figure 9 shows that local Nusselt number is increased i.e. heat transfer enhanced, with positive $\varepsilon$ and depressed with negative $\varepsilon$ principally near the cone apex. As we progress along the cone surface from the apex, the influence of $\varepsilon$ is consistently reduced, with profiles soon merging. Joshi and Gebhart [40] also showed that the pressure work parameter enhances heat transfer from surfaces for lower Prandtl numbers, as is apparent from figure 9 in our study.

Figures 10-13 present the response of skin friction $\left(C_{f x} G r_{x}^{1 / 4}\right)$, local Nusselt number $\left(N u_{x} G r_{x}^{=1 / 4}\right)$, velocity $\left(F^{\prime}\right)$ and temperature $(G)$ to a change in local Grashof number $\left(G r_{x}\right)$. $G r_{x}=\frac{g \beta \cos \gamma\left[T_{w}-T_{\infty}\right] x^{3}}{v^{2}}$ and effectively this parameter simulates the relative effect of buoyancy force (free convection) to viscous force in the regime. With increasing $G r_{x}$ there is a distinct escalation in skin friction (figure 10) at the cone surface indicating that buoyancy forces aid the momentum development i.e. accelerate the flow. Maximum values of skin friction arise in all cases very close to the cone apex. Local Nusselt number function (figure 11) is also found to be enhanced slightly with a rise in $G r_{x}$. The greatest influence occurs at the cone apex region with progressively less effect further along the cone surface. Increasing buoyancy therefore assists in enhancing cone surface heat transfer rate in the vicinity of the leading edge. Figure 12 in concurrence with figure 10 demonstrates that velocity is boosted considerably with increasing local Grashof number, since the elevation in buoyancy force serves to accelerate the flow both along the cone surface and into the boundary layer regime. A velocity peak close to the cone surface is observed at $\eta \sim 1$ and this peak migrates further from the wall (cone surface) with 
increasing $G r_{x}$. In figure 13 we observe that temperature, $G$, is diminished with increasing $G r_{x}$ throughout the boundary layer regime transverse to the cone surface. Since buoyancy forces enhance heat transfer rates (figure 11), greater heat quantities will be conducted from the fluid to the wall with increasing $G r_{x}$ which will serve to cool the boundary layer and induced a fall in temperatures.

In figure 14 the combined influence of local Hartmann numbers $\left(H a_{x}\right)$ and pressure work parameters $(\varepsilon)$ on skin friction is plotted. It is interesting to note that the hydromagnetic drag force exerts a much more prominent effect than pressure work. With $H a_{x}=0$, there is a clear separation in skin friction profiles, with the trend agreeing with figure 8 i.e. skin friction is enhanced with negative $\varepsilon$ and decreased with positive $\varepsilon$. Figure 8 was computed with $H a_{x}=1, \varepsilon$ $=0, n=0.4, G r_{x}=10, \operatorname{Pr}=0.72$. However with much stronger magnetic field in figure 14 than figure 8, i.e. $H a_{x}=5.0$, we observe that while skin friction is clearly substantially lower than for the non-conducting case $\left(H a_{x}=0\right)$, the influence of $\varepsilon$ is very small; infact the pressure work effect is really only discernible near the cone apex. As such the hydromagnetic effect is much more powerful than the pressure (stress) work effect in the present flow domain.

Figure 15 shows the combined influence of local Hartmann numbers $\left(H a_{x}\right)$ and pressure work parameters $(\varepsilon)$ on local Nusselt number function. As in figure $9, N u_{x} G r_{x}^{-1 / 4}$ is increased for positive $\varepsilon$ and lowered with negative $\varepsilon$ mainly near the cone leading edge ( $\xi=0)$. Also as found in figure 5, $N u_{x} G r_{x}^{-1 / 4}$, is slightly reduced with increasing $H a_{x}$ to 5.0- again the heat transfer rate at the cone surface is greater for the non-conducting case $\left(H a_{x}=0\right)$, for any value of $\varepsilon$.

Figures 16 and 17 show the composite effects of local Hartmann number $\left(H a_{x}\right)$ and temperature power law index parameters $(n)$ on local Nusselt number function. The dominant effect of applied magnetic field i.e. local Hartmann number on skin fiction (figure 16) is apparent once again. The influence of $n$ is clearly much less effective on the skin friction than applied magnetic field, the latter exerting as shown earlier in figure 8, a strongly inhibiting effect on flow whereas the former has a much weaker inhibiting effect. In figure 17, the beneficial influence on $N u_{x} G r_{x}^{-1 / 4}$ of increasing $n$ is again evident as is the negative influence of local Hartmann number, the latter again serving to depress cone surface heat transfer rates.

Finally in figures 18 and 19 we have plotted the collective effects of pressure work parameter $(\varepsilon)$ and temperature power law index parameters $(n)$ on skin friction and surface heat transfer gradient (local Nusselt number). Again we observe that skin friction (figure 18) is greater for the isothermal case (as documented earlier) compared with the non-isothermal scenario, and that negative pressure work parameter enhances skin friction whereas positive values depress skin friction i.e. retard the flow. The influence of $n$ is clearly greater however than $\varepsilon$. Figure 19 
confirms the counter-productive influence of negative $\varepsilon$ on $N u_{x} G r_{x}^{-1 / 4}$ and the enhancing influence of positive $\varepsilon$. Local heat transfer gradient $N u_{x} G r_{x}^{-1 / 4}$ is again shown to be boosted for the nonisothermal case $(n=0.8)$ as compared with the isothermal case $(n=0)$.

\section{CONCLUSIONS}

A mathematical model has been presented for the laminar, magneto-convection gas boundary layer flow past a cone with variable surface temperature and pressure work effects. The velocity and thermal boundary layer equations have been non-dimensionalized using a pseudo-similarity transformation and a number of special cases of the general model described. NSM has been elected to solve the boundary value problem for a wide range of the governing parameters, with comparisons made against previous non-magnetic and non-transpiring conical flows. The effects of local Hartmann number, pressure work, thermal power-law exponent, local Grashof number and Prandtl number on skin friction and local Nusselt number distributions have been presented graphically and discussed in detail. The computations show that with increasing power law index (non-isothermal effect), skin friction at the cone is reduced whereas heat transfer rate is increased. Skin friction is elevated with negative pressure work parameter whereas it is reduced with positive values. Increasing local Hartmann number reduces cone surface heat transfer rates and also significantly decelerates the boundary layer flow i.e. reduces skin friction. With increasing local Grashof number i.e. stronger thermal buoyancy, skin friction is enhanced whereas temperatures are reduced. The study finds immediate applications in magneto-gas dynamic heat transfer in astronautics, materials processing and geophysical magneto-fluid dynamics. Currently the authors are extending this study with a finite element model [42-44] to include the effects of oblique magnetic field [45], Hall currents and magnetic induction [46]. Furthermore the use of surface injection of nanofluids to cool the cone surface [47] is being explored. In this regard an interesting extension to the current simulations is non-Newtonian nanofluid convection which has been addressed in detail by Prasannakumara et al. [48, 49] using various rheological constitutive models. These developments will also be investigated in the near future.

\section{ACKNOWLEDGEMENTS}

Dr. O. Anwar Bég is grateful to the late Dr. Howard Brenner, formerly Willard Dow Professor of Chemical Engineering at MIT, USA for providing some excellent guidance on pressure work and 
dissipation fluid mechanics aspects. All the authors are also grateful to the reviewers for their constructive comments which have served to improve the present investigation.

\section{REFERENCES}

[1] Witte, A.B. and Harper, E.Y., Experimental investigation and empirical correlation of local heat transfer rates in rocket-engine thrust chambers, Tech. Report 32-344, Jet Propulsion Laboratory, California Institute of Technology, Pasadena, California, March (1962).

[2] Medford, J.E., Transient radial heat transfer in uncooled rocket nozzles, Aerospace Engineering, 21, 10, 15-21 (1962)

[3] Chamkha, A.J. and M. A. Quadri, Combined heat and mass transfer by hydromagnetic natural convection over a cone embedded in a non-Darcian porous medium with heat generation/absorption effects, Heat and Mass Transfer, 38, 487-495 (2002).

[4] Bartz, D.R., A simple equation for rapid estimation of rocket nozzle convective heat transfer coefficients, Jet Propulsion, 37, 1, 49-51 (1957).

[5] Streif, M. L., Heat transfer to a cone segment model, General Dynamics and Astronautics, Report, San Diego, California, July (1960).

[6] Braun, W.H., Ostrach, S., Heighway, J.E., Free convection similarity flows about twodimensional and axisymmetric bodies with closed lower ends, Int. J. Heat Mass Transfer, 21, 1-2, 121-135 (1961).

[7] Hering, R.G. and Grosh, R.J., Laminar free convection from a non-isothermal cone, Int. J. Heat Mass Transfer, 5, 11, 1059-1068 (1962).

[8] Hering, R.G., Laminar free convection from a non-isothermal cone at low Prandtl numbers, Int. J. Heat Mass Transfer, 8, 10, 1333-1337 (1965).

[9] Roy, S., Free convection over a slender vertical cone at high Prandtl numbers, ASME J. Heat Transfer, 96, 1-4, 174-176 (1974).

[10] Na, T.Y. and Chiou, J. P., Laminar natural convection over a slender vertical frustrum of a cone, Heat Mass Transfer, 12, 2, 83-87 (1979).

[11] Lin, F.N., Laminar free convection from a vertical cone with uniform surface heat flux, Letters in Heat and Mass Transfer, 3, 1, 49-58 (1976).

[12] Alamgir, M., Overall heat transfer from vertical cones in laminar free convection: an approximate method, ASME J. Heat Transfer, 101, 1-4, 174-176 (1979).

[13] Pop, I. and Takhar, H.S.: Compressibility effects in laminar free convection from a vertical cone. Applied Scientific Research, 48, 71- 82 (1991). 
[14] Hossain, M.A. Munir, M.S. and Takhar, H.S.: Natural convection flow of a viscous fluid about a truncated cone with temperature dependent viscosity. Acta Mechanica. 140, 171-181 (2000).

[15] Hossain, M.A. and Paul, S.C., Free convection from a vertical permeable cone with nonuniform surface temperature, Acta Mechanica, 151, 1-2, 103-114 (2001).

[16] Takhar, H.S., A.J. Chamkha and G. Nath, Effect of variable thermophysical quantities on the natural convection flow of gases over a vertical cone, Int. J. Engineering Science, 42, 243-256 (2003).

[17] Chamkha, A.J., H.S. Takhar and G. Nath, Unsteady compressible boundary layer flow over a circular cone near a plane of symmetry, Heat and Mass Transfer, 41, 632-641 (2005).

[18] Roy, S., Datta, P. and Mahanti, N.C., Non-similar solution of an unsteady mixed convection flow over a vertical cone with suction/injection, Int. J. Heat Mass Transfer, 50, 1-2, 181-187 (2007).

[19] Singh, P.J. and S. Roy, Unsteady mixed convection flow over a vertical cone due to impulsive motion, Int. J. Heat and Mass Transfer, 50, 5/6, 949-959 (2007).

[20] Kumari, M. and Nath, G., Transient laminar compressible boundary layers over a permeable circular cone near a plane of symmetry, Int. J. Heat Mass Transfer, 48, 13, 2771-2778 (2005).

[21] Sluyter, M. M. and Touryan, K. J., The effect of a magnetic field on a rotating cone in compressible flow, Sandia National Labs Report, New Mexico, USA, AIAA-1969-721 (1969).

[22] Surma Devi, C.D., Takhar. H.S. and Nath, G., MHD flow past a cone, Acta Technica Csav, 31, 400-409 (1986).

[23] Singh, S.N., Takhar. H.S. Int. J. Ram, P.C.: Magnetohydrodynamic flow between coaxial rotating cone and a cylinder under the influence of radial a magnetic field. $J$. Magnetohydrodynamics and Plasma Research, 6, 21-32 (1996).

[24] Chamkha, A.J., Non-Darcy hydromagnetic free convection from a cone and a wedge in porous media, Int. Comm. Heat and Mass Transfer, 23, 875-887 (1996).

[25] Chamkha, A.J., A.-R.A. Khalid and O. Al-Hawaj, Simultaneous heat and mass transfer by natural convection from a cone and a wedge in porous media, J. Porous Media, 3, 155-164, (2000).

[26] Chamkha, A.J., Coupled heat and mass transfer by natural convection about a truncated cone in the presence of magnetic field and radiation effects, Numerical Heat Transfer, Part A, 39, pp. 511-530 (2001). 
[27] Chamkha, A.J. and A. Al-Mudhaf, Unsteady heat and mass transfer from a rotating vertical cone with a magnetic field and heat generation or absorption effects, Int. J. Thermal Sciences, Volume 44, pp. 267-276, 2005.

[28] Alam, M. M., Alim, M. A. and Chowdhury, M. M. K., Free convection from a vertical permeable circular cone with pressure work and non-uniform surface temperature, Nonlinear Analysis: Modelling and Control, 12, 1, 21-32 (2007).

[29] Gebhart, B., Effects of viscous dissipation in natural convection, J. Fluid Mechanics, 14, 2, 225-232 (1962).

[30] Takhar, H.S. and Soundalgekar, V.M.: Effects of viscous dissipation and stress work on heat transfer in a boundary layer flow past a semi-infinite horizontal flat plate. " $L$ " Aerotechnica Missili E. Spazio. 56, 210-212 (1978).

[31] Soundalgekar, V.M., Takhar. H.S. and Vignesam, N.V.: Combined free and forced convection flow past a semi-infinite vertical plate with variable surface temperature. Nuclear Engineering and Design. 110, 95-98 (1988).

[32] Soundalgekar, V.M., Murty, T.V.R. and Takhar, H.S.: Heat transfer in MHD unsteady stagnation point flow with a variable temperature. Indian J. Pure and Applied Math. 21, 384-389 (1990).

[33] Ganesan,P., Ekambavanan, K., Soundalgekar, V.M. and Takhar, H.S.: Transient free convection past a semi-infinite vertical plate with variable surface temperature. Int. J. Num. Meth. Heat and Fluid Flow, 7, 186-202 (1997).

[34] Landau, L.D. and Lifschitz, E., Electrodynamics of Continuous Media, International Course in Theoretical Physics, Pergamon, Oxford (1959).

[35] Bég, O. Anwar, J. Zueco, R. Bhargava, H.S. Takhar, Magnetohydrodynamic convection flow from a sphere to a non-Darcian porous medium with heat generation or absorption effects: network simulation, Int. J. Thermal Sciences, 48, 5, 913-921 (2009).

[36] Bég, O. Anwar, J. Zueco, M. Norouzi, M. Davoodi, A. A. Joneidi, Assma F. Elsayed, Network and Nakamura tridiagonal computational simulation of electrically-conducting biopolymer micro-morphic transport phenomena, Computers in Biology and Medicine, 44, 44-56 (2014).

[37] Pspice 6.0. (1994) Irvine, California 92718. Microsim Corporation, 20 Fairbanks

[38] Cramer, K.C. and Pai, S.I., Magnetofluid Dynamics for Engineers and Applied Physicists, MacGraw-Hill, New York (1973). 
[39] Hill, P.G. and Peterson, C.R., Mechanics and Thermodynamics of Propulsion, AddisonWesley Series in Aerospace Science, H.W. Emmons and S.S. Penner (Editors), Reading, Massachusetts, USA, $2^{\text {nd }}$ edition (1967).

[40] Joshi, Y. and Gebhart, B., Effect of pressure stress work and viscous dissipation in some natural convection flows, Int. J. Heat and Mass Transfer, 24, 1577-1588 (1981).

[41] Ackroyd, J.A.D., Stress work effects in laminar flat-plate natural convection, J. Fluid Mechanics, 62, 677-695 (1974).

[42] S. Rawat, R. Bhargava, Renu Bhargava and O. Anwar Bég, Transient magneto-micropolar free convection heat and mass transfer through a non-Darcy porous medium channel with variable thermal conductivity and heat source effects, Proc.IMechE Part C- J. Mechanical Engineering Science, 223, 2341-2355 (2009).

[43] R. Bhargava, S. Sharma, O. Anwar Bég and Zueco, J, Finite element study of nonlinear twodimensional deoxygenated biomagnetic micropolar flow, Comm. Nonlinear Science and Numerical Simulation, 15, 1210-1233 (2010).

[44] Puneet Rana, R. Bhargava and O.Anwar Bég, Numerical solution for mixed convection boundary layer flow of a nanofluid along an inclined plate embedded in a porous medium, Computers and Mathematics with Applications, 64, 2816-2832 (2012).

[45] O. Anwar Bég, S.K. Ghosh and M. Narahari, Mathematical modelling of oscillatory MHD Couette flow in a rotating highly permeable medium permeated by an oblique magnetic field, Chemical Engineering Communications, 198, 235-254 (2010).

[46] S K. Ghosh, O. Anwar Bég and A. Aziz, A mathematical model for magnetohydrodynamic convection flow in a rotating horizontal channel with inclined magnetic field, magnetic induction and Hall current effects, World J. Mechanics,1, 137-154 (2011).

[47] O. Anwar Bég and D. Tripathi Mathematica simulation of peristaltic pumping with doublediffusive convection in nanofluids: a bio-nano-engineering model, Proc. IMechE Part N - J. Nanoengineering and Nanosystems, 225, 99-114 (2012).

[48] B. C. Prasannakumara, M. R. Krishnamurthy, B. J. Gireesha, and Rama S. R. Gorla, Effect of multiple slips and thermal radiation on MHD flow of Jeffery nanofluid with heat transfer, $J$. Nanofluids, 5, 82-93 (2016).

[49] Prasannakumara, B., Gireesha, B., Gorla, R., and Krishnamurthy, M., Effects of chemical reaction and nonlinear thermal radiation on Williamson nanofluid slip flow over a stretching sheet embedded in a porous medium, ASCE J. Aerospace Engineering (2016). 10.1061/(ASCE)AS.1943-5525.0000578, 04016019. 